\title{
Regional Differences in Information Privacy Concerns After the Facebook-Cambridge Analytica Data Scandal ${ }^{\dagger}$
}

\author{
Felipe González-Pizarro ${ }^{1^{\dagger}}\left(\mathbb{0}\right.$, Andrea Figueroa $^{2}$, Claudia López $^{1^{*}}$ (D) \& Cecilia \\ Aragon $^{2}$ \\ ${ }^{1}$ Departamento de Informática, Universidad Técnica Federico Santa María, Chile \\ (felipe.gonzalezp.12@sansano.usm.cl, claudia@inf.utfsm.cl)²Human-Centered Data Science Lab, \\ University of Washington, Seattle, United States (afigue@uw.edu, aragon@uw.edu) ${ }^{\dagger}$ Department of \\ Computer Science, University of British Columbia, Vancouver, Canada (felipegp@cs.ubc.ca)
}

\begin{abstract}
.
While there is increasing global attention to data privacy, most of their current theoretical understanding is based on research conducted in a few countries. Prior work argues that people's cultural backgrounds might shape their privacy concerns; thus, we could expect people from different world regions to conceptualize them in diverse ways. We collected and analyzed a large-scale dataset of tweets about the \#CambridgeAnalytica scandal in Spanish and English to start exploring this hypothesis. We employed word embeddings and qualitative analysis to identify which information privacy concerns are present and characterize language and regional differences in emphasis on these concerns. Our results suggest that related concepts, such as regulations, can be added to current information privacy frameworks. We also observe a greater emphasis on data collection in English than in Spanish. Additionally, data from North America exhibits a narrower focus on awareness compared to other regions under study. Our results call for more diverse sources of data and nuanced analysis of data privacy concerns around the globe.
\end{abstract}

Keywords: Online privacy; Twitter; Word embedding; Content analysis; IUIPC

\section{Introduction}

The right to control one's personal information has gained significant importance lately (Lee et al., 2019). Indeed, 58\% of the countries have data protection and privacy legislation, while another $10 \%$ have drafted legislation about it (United Nations Conference on Trade and Development, 2020). This broad interest is related to the massive amount of personal data collected by information systems and the risk that such information could be wrongly distributed online (Lee et al., 2019).

The study of information privacy has advanced our understanding of individuals' concerns regarding organizational practices associated with collecting and using their personal information (Smith et al., 1996). However, a literature review revealed a strong bias towards USA-centered studies across privacy concerns literature and warned about the limitations to generalizability this entails (Bélanger and Crossler, 2011; Okazaki et al., 2020). The review's authors hypothesized that individuals from different world regions have diverse cultures, values, and laws, which can, in turn, result in different conceptualizations of information privacy and its impacts (Bélanger and Crossler, 2011; Mohammed and Tejay, 2017). To study these differences, privacy research has often relied on survey-based studies (Cockcroft and Rekker, 2016). For example, a questionnaire was applied to explore differences in privacy perceptions between Facebook users from Germany and the USA (Krasnova and Veltri, 2010), and a cross-national survey was conducted to evaluate information attitudes of

$\dagger$ This version corresponds to the accepted manuscript (29 November 2021). For the final published version and citation please see https://doi.org/10.1007/s10606-021-09422-3 
consumers in the USA and Brazil (Markos et al., 2017). These multi-country privacy studies have had limited sample sizes, which makes the results difficult to generalize (Lee et al., 2019; Huang and Bashir, 2016). They also tend to be focused on one or two cultures, usually including the USA (Cockcroft and Rekker, 2016). Hence, multicountry information privacy research is still needed to extend our understanding of this increasingly relevant topic around the globe (Adu et al., 2019; Zou et al., 2018).

We propose an alternative approach to study information privacy concerns over a large geographical scope. This work combines word embeddings, open coding, and content analysis to examine tweets related to a large data breach scandal. We seek to characterize similarities and differences in privacy terms across people who tweet about this issue in different languages and from different world regions. Inspired by (Rho et al., 2018), where text analysis was used to analyze answers about individuals' privacy concerns, we analyze the semantic context in which privacy-related terms were used in tweets written by different groups of people.

We focus on the Facebook-Cambridge Analytica data scandal. In 2018, the firm Cambridge Analytica was accused of collecting and using the personal information of more than 87 million Facebook users without their authorization (Venturini and Rogers, 2019; Isaak and Hanna, 2018; Lapaire, 2018). The scandal sparked multiple conversations over technology's societal impact and risks to citizens' privacy and well-being worldwide. Opinions, facts, and stories related to it took place on different social media platforms such as Twitter, where the hashtag \#DeleteFacebook became a trending topic for several days (Lin, 2018; Mirchandani, 2018).

We analyze more than a million public tweets in Spanish or English that use hashtags or keywords related to the scandal. We divide the dataset by language (Spanish and English) and regions (Latin America, Europe, North America, and Asia) and create word-embeddings for each subset. Then, we systematically analyze and compare the semantic context of four keywords, such as data, privacy, user, and company, across the embeddings. We contrast our results with one of the most used information privacy concerns framework to find terms and tweets matching different concerns. Then, we test a null hypothesis that there is no difference in emphasis on information privacy terms across languages and world regions. In this process, we discover the presence of related concepts that could be integrated into information privacy frameworks, such as regulations. We also observe statistically significant language differences in emphasis on data collection and significant regional differences in emphasis on awareness. Finally, we discuss the implications of our results.

We summarize prior work on information privacy concerns in Section 2. Section 3 introduces our research question and hypothesis. Section 4 details our research method, while Section 5 reports on our findings. Section 6 offers a discussion of our results, limitations, and future work. Finally, Section 7 provides our conclusions.

\section{Information privacy concerns}

Information privacy concerns emerge when an individual "feels threatened by a perceived unfair loss of control over their privacy by an information-collecting body" (Lee et al., 2015). Previous research argues that information privacy concerns are a multidimensional construct(Jozani et al., 2020; Correia and Compeau, 2017; Heravi et al., 2018). A multidimensional approach allows identifying to what extent users are concerned about different aspects of information privacy (Yun et al., 2019; Hong and Thong, 2013; Hong and Thong, 2013). Different authors have proposed alternative 
conceptualizations to measure information privacy concerns. We briefly review the most adopted ones in the following subsection. Then, we summarize prior work on differences in privacy concerns across countries, regions, and other characteristics.

Prior research has examined privacy concerns from other perspectives as well (Heravi et al., 2018; Gerber et al., 2018; Yun et al., 2019). A vast portion of privacy research on social networking sites has focused on examining users' privacy behaviors, such as the intention to provide personal information or transact online (Wisniewski et al., 2017; Kokolakis, 2017; Gerber et al., 2018; Oghazi et al., 2020; Chai, 2020; Heravi et al., 2018; Markos et al., 2017). In a similar direction, several studies have investigated the use of privacy setting configurations (Wisniewski et al., 2015; Vitak et al., 2015; Wisniewski et al., 2017; Li et al., 2020). Rather than centering on behavior or behavioral intention, we focus our review on information privacy concerns that characterize general personal dispositions (C. Sipior et al., 2013). We think that this part of the literature aligns better with what people can say, in a declarative way, about data privacy on social media.

\subsection{Assessing INFORMATION PRIVACY CONCERNS}

Two questionnaires have been widely used to evaluate individuals' information privacy concerns (Bélanger and Crossler, 2011; Cockcroft and Rekker, 2016; Morton and Sasse, 2014): Concerns for Information Privacy (CFIP) and Internet Users' Information Privacy Concerns (IUIPC).

\subsubsection{CFIP: Concerns for Information Privacy}

The CFIP framework (Smith et al., 1996) focuses on individuals' perceptions of how organizations use and protect personal information (Van Slyke et al., 2006). CFIP identifies four dimensions:

- Collection: concerns about personal data that is collected over time;

- Unauthorized secondary use: concerns about organizations using personal data for another purpose without the individual's authorization;

- Improper access: concerns about unauthorized people having access to personal data;

- Errors: concerns about adequate protections from deliberate and accidental errors in personal data.

To measure them, Smith et al. (1996) proposed and validated a 15-item questionnaire. The CFIP questionnaire was validated by surveying 355 consumers from the USA and applying confirmatory factor analysis (CFA) (Stewart and Segars, 2002). So far, this questionnaire had been considered as one of the most established methods to measure quantitatively information privacy concerns (Harborth and Pape, 2018) and had been widely used in the literature (Harborth and Pape, 2017; Stewart and Segars, 2002). However, the CFIP and its measurement instrument were originally defined for users in an offline context (Palos-Sanchez et al., 2017). As the Internet enabled new ways to collect and process data, it was expected that new concerns about information privacy might emerge (Malhotra et al., 2004), and a new framework was proposed: the IUIPC. 


\subsubsection{IUIPC: Internet Users' Information Privacy Concerns}

Malhotra et al. (2004) introduced the IUIPC framework and conceptualized Internet users' concerns about information privacy from a perspective of fairness. Drawing from social contract theory (C. Sipior et al., 2013), Malhotra et al. (2004) argue that personal data collection is perceived to be fair when a user has control over their personal data and is informed about the intentions that organizations have about how to use it. The IUIPC includes three constructs:

- Collection: concerns about the amount of personal data owned by others compared to the perceived benefits (Malhotra et al., 2004). It is related to the perceived fairness of the outcomes one receives. Users provide information if they expect to obtain something of value after a cost-benefit analysis of a transaction.

- Control: concerns about control over personal information, including approval, modification of collected data, and opportunity to opt-in or opt-out from data collection (Malhotra et al., 2004). It is related to the perceived fairness of the procedures that maintain personal data.

- Awareness: concerns about personal awareness of organizational information practices (Malhotra et al., 2004). It relates to issues of transparency of the procedures and specificity of information to be used.

A 10-item questionnaire to assess these constructs was validated in (Malhotra et al., 2004). The questionnaire has been widely used to this day (Yun et al., 2019; Raber and Krüger, 2018) because it considers the Internet context, and it can explain more variance in a person's willingness to transact than CFIP (Rowan and Dehlinger, 2014). Recent work has explored text mining as an alternative research method to identify IUIPC dimensions. Raber and Krüger (2018) found that IUIPC dimensions can be derived from written text. They observed a correlation between IUIPC concerns, as measured by the questionnaire, and LIWC language features of social media posts from a sample of 100 users.

\subsubsection{Other instruments of assessment}

The Westin-Harris Privacy Segmentation Index measures individuals' attitudes and concerns about privacy and how they vary over time (Kumaraguru and Cranor, 2005) based on answers to three questions (Egelman and Peer, 2015a; Woodruff et al., 2014). It categorizes individuals into three groups (Kumaraguru and Cranor, 2005; Da Veiga, 2018; Motiwalla et al., 2014): Fundamentalists are highly concerned about sharing their data, protect their personal information, prefer privacy controls over consumer-service benefits, and are in favor of new privacy regulations; Pragmatists tend to seek a balance between the advantages and disadvantages of sharing personal information before arriving at a decision; Unconcerned users believe there is a greater benefit to be derived from sharing their personal information, trust organizations that collect their personal data and are the least protective of their privacy.

The Westin-Harris' index was introduced as a way to meaningfully classify internet users based on their attitude toward privacy and their motivations to disclose personal information (Torabi and Beznosov, 2016). It has been used for several decades. However, recent studies have raised questions about its validity (Egelman and Peer, 2015a). Prior work has failed to establish a significant correlation between the Westin-Harris' segmentation and context-specific, privacy-related actual or intended behaviors (Consolvo et al., 2005; Woodruff et al., 2014; Egelman and Peer, 2015b). 
The existence of a mismatch between privacy concerns and privacy behaviors, known as the "privacy paradox" (Kokolakis, 2017; Dienlin and Trepte, 2015), motivated the creation of a new measurement instrument. Buchanan's Privacy Concern scale aims to capture different aspects of the paradox. Buchanan et al. (2007) developed three privacy scales: two of them assess privacy behavior, and the third one measures information privacy concerns. However, some limitations have been identified. Their scales are not able to identify different privacy dimensions, but only one, which appears to map onto the general concept of privacy concern. Thus, a more fine-grained examination is desirable to improve the design of this scale (Buchanan et al., 2007).

Because our study focuses on people's comments about a specific information privacy scandal (and not their privacy behavior), our work will mostly build upon the information privacy concerns frameworks, particularly the IUIPC.

\subsection{DiffERENCES ON INFORMATION PRIVACY CONCERNS}

Information privacy concerns can vary across individuals based on peoples' perceptions and values (Buchanan et al., 2007). People may have different concerns even if they experience the same situation (Lee et al., 2015). It has been argued that information privacy concerns can be influenced by different factors (Smith et al., 2011), such as national culture (Cho et al., 2009; Huang and Bashir, 2016; Cao and Everard, 2008; Krasnova and Veltri, 2010), and individuals' demographics (e.g., age, gender) (Zukowski and Brown, 2007; Lee et al., 2019; Jai and King, 2016; Rowan and Dehlinger, 2014; Cho et al., 2009; Markos et al., 2017). We review these factors below.

\subsubsection{National Culture}

While there are similarities in what privacy means across cultures (Cockcroft and Rekker, 2016), there is no universal consensus on its definition (Cannataci, 2009). According to Newell (1995), several cultures do not possess an equivalent term to the English' privacy definition in their own language, e.g., Arabic, Dutch, Japanese, and Russian. Nevertheless, this does not mean that these cultures lack a sense of privacy (Newell, 1995). Every society appreciates privacy in some way, but the expression of it varies (Cho et al., 2009).

The concept of national culture has been studied as one of the factors related to information privacy concerns (Nov and Wattal, 2009; Malhotra et al., 2004; Bellman et al., 2004). National culture can be defined as "the collective mindset distinguishing the member of one nation from another" (Cho et al., 2009). Hofstede's cultural dimensions theory (Hofstede, 1983) has been the most used conceptual model to study cultural differences in this context. This trend is expected since Hofstede's theory has been widely used to study the relationship between culture and technology (Leidner and Kayworth, 2006), even though there are a number of criticisms of this theory (Terlutter et al., 2006). The latest version of this theory proposes six cultural dimensions (Hofstede, 2011). Among them, the individualism/collectivism dimension has been found relevant to information privacy concerns. Individualism/collectivism refers to the extent to which individuals are part of groups beyond their immediate families.

Differences in information privacy concerns have been explained using some cultural dimensions at a country and regional level (see Table I). Participants from individualistic countries (Australia and United States) exhibited a higher level of 
online privacy concerns than individuals from collectivist countries (Cho et al., 2009). The authors' rationale is that high individualism is associated with an emphasis on private life and independence from the collective; thus, people from individualist countries are more worried about privacy intrusions. In the same direction, Bellman et al. (2004) found that controlling for internet experience and privacy regulations, people from countries with high individualism show deeper concern about two CFIP dimensions: unauthorized secondary use and improper access.

On the other hand, no regional differences in privacy concerns were found through online surveys with 226 English-fluent crowd workers from six regions (Africa, Asia, Western Europe, Eastern Europe, North America, and Latin America). The authors argued that it is unclear if their finding is due to true similarities or a lack of enough power in measuring privacy concerns (Huang and Bashir, 2016).

Table I. Culture and information privacy concerns

\begin{tabular}{|c|c|c|c|}
\hline $\begin{array}{l}\text { Independent } \\
\text { variables }\end{array}$ & Method & $\begin{array}{l}\# \text { participants } \\
\text { and origin }\end{array}$ & Key findings \\
\hline $\begin{array}{l}\text { National } \\
\text { culture }\end{array}$ & $\begin{array}{l}\text { 5-item questionnaire (Cho et al., } \\
\text { 2009), based on a unidimensional } \\
\text { conceptualization of online privacy } \\
\text { concerns. Items were comprehen- } \\
\text { sive enough to measure general } \\
\text { concerns about online privacy. }\end{array}$ & $\begin{array}{l}1261 \text { from Seoul, } \\
\text { Singapore, } \\
\text { Bangalore, } \\
\text { Sydney, New } \\
\text { York }\end{array}$ & $\begin{array}{l}\text { Participants from } \\
\text { individualistic countries } \\
\text { exhibited higher concern } \\
\text { about online privacy } \\
\text { (Cho et al., 2009) }\end{array}$ \\
\hline $\begin{array}{l}\text { National } \\
\text { culture }\end{array}$ & $\begin{array}{l}\text { 15-item questionnaire (CFIP) } \\
\text { (Smith et al., 1996), based on } \\
\text { a multidimensional constructive } \\
\text { model of privacy concerns (collec- } \\
\text { tion, unauthorized secondary use, } \\
\text { improper access, errors). }\end{array}$ & $\begin{array}{l}534 \text { from } 38 \\
\text { countries }\end{array}$ & $\begin{array}{l}\text { Participants } r \text { from } \\
\text { individualistic countries } \\
\text { showed higher concern } \\
\text { about improper access } \\
\text { and secondary use } \\
\text { (Bellman et al., 2004) }\end{array}$ \\
\hline $\begin{array}{l}\text { Regional } \\
\text { culture }\end{array}$ & $\begin{array}{l}\text { 4-item questionnaire (Dinev and } \\
\text { Hart, 2006), based on a unidimen- } \\
\text { sional conceptualization of privacy } \\
\text { concerns, which is defined as ap- } \\
\text { prehension about how online per- } \\
\text { sonal information is used by others. }\end{array}$ & $\begin{array}{lr}226 \text { from } & \text { Africa, } \\
\text { Asia, Western } \\
\text { and } & \text { Eastern } \\
\text { Europe, } & \text { North } \\
\text { and } & \text { Latin } \\
\text { America } & \end{array}$ & $\begin{array}{l}\text { No regional differences } \\
\text { in privacy concerns } \\
\text { were found (Huang and } \\
\text { Bashir, 2016) }\end{array}$ \\
\hline
\end{tabular}

\subsubsection{Language}

Relatedly, the Sapir-Whorf hypothesis suggests that the structure of anyone's native language influences the world-views they will acquire (Kay and Kempton, 1984). Depending on the language, a message is coded and decoded differently based on standardized language norms and culture (Zarifis et al., 2019). Thus, individuals who speak different native languages could think, perceive reality and organize the world around them in different ways (Hussein, 2012).

Previous work has explored how user-generated content can reveal different views about the same issues among people who write in different languages. Jiang et al. (2017) conducted a semantic network analysis to examine the semantic differences that emerge from the Wikipedia articles about China. Results suggest that Chinesespeaking and English-speaking contributors framed articles about China in different and even opposite ways, which were aligned to their national cultures and values. The Chinese version framed them from perspectives of authority respect, emphasizing 
harmony and patriotism. Articles in English were written from the point of view that is distinctive of many Western societies: the core value of democracy.

A potential role of the spoken language in the information privacy context has also been studied. Li et al. (2017) created a cross-cultural privacy prediction model. The model applies supervised machine learning to predict users' decisions on the collection of their personal data. Using answers from an online survey of 9,625 individuals from 8 countries on four continents: Canada, China, Germany, United States, United Kingdom, Sweden, Australia and, India,they found that the model's prediction accuracy improved when adding individual's language (English, Chinese, French, Swedish, and German) or Hofstede's cultural dimensions. Our work will build upon this line of reasoning to deepen our understanding of information privacy concerns across the globe.

\subsubsection{Other individual characteristics}

Even though our work will not address the relationship between demographics and information privacy concerns, we will briefly review the literature about this topic.

Prior studies suggest that older Internet users are more concerned about online information privacy than younger ones (Cho et al., 2009). Older participants were more sensitive to privacy issues and exhibited a greater desire to control the amount of information collected about them (Zukowski and Brown, 2007). In contrast, younger users declared themselves to be more willing to share their personal information with third parties (Jai and King, 2016).

The relation between privacy concerns and gender has also been studied (Cho et al., 2009). Jai and King (2016) found that women were less willing than men to permit third parties to share their personal information. Similarly, Rowan and Dehlinger (2014) observed that women reported greater information privacy concerns than their male counterparts. Both studies considered gender as binary.

Another relevant factor is participants' internet experience. As users grow in internet experience, concerns for online information privacy may decrease (Zukowski and Brown, 2007). Bellman et al. (2004) concluded that participants with more internet experience were less concerned about online privacy overall, and in particular, were less worried about improper access and secondary use. This could be explained by increased familiarity with online privacy practices (Zukowski and Brown, 2007).

\section{Research Questions}

Overall, while concepts around information privacy concerns have been extensively investigated, some limitations are shared among the studies that assess differences in these concerns worldwide. Most research has been conducted through surveys and has focused only on a few geographic regions, with a notable exception of ( $\mathrm{Li}$ et al., 2017). Many studies have had a limited sample size (Vitkauskaite, 2010; Ur and Wang, 2013; Ebert et al., 2020; Chai, 2020; Li et al., 2020; Oghazi et al., 2020; Krasnova and Veltri, 2010); thus, their findings' generalizability has been questioned (Lee et al., 2019). Moreover, when information privacy concerns questionnaires are delivered in English to speakers of other languages, key differences among countries may be obscured, as has happened with other cross-national research (Harzing and Maznevski, 2002; Harzing, 2006). Unfortunately, conducting larger-scale, multicountry, and multi-language surveys can be quite expensive (Harzing, 2005; Li et al., 2020). Yet, large-scale research to deepen our understanding of information privacy 
concerns worldwide is still needed (Vitkauskaite, 2010; Chai, 2020; Li et al., 2020; Zou et al., 2018; Oghazi et al., 2020; Okazaki et al., 2020).

We seek to assess the feasibility of using social media data to identify information privacy concerns and characterize language and regional differences. Twitter is a popular micro-blogging service where individuals from different world regions who speak diverse languages share opinions, information, and experiences (Yaqub et al., 2017; Shen and Kuo, 2014). Mining text from this platform has been used as a fast and inexpensive method to gather opinions from individuals (O'Connor et al., 2010), which can complement findings obtained from traditional polls or other research methods. Prior research has found a significant correlation between tweets and public opinion in diverse domains (O'Connor et al., 2010; Tumasjan et al., 2010; Ilyas et al., 2020; Tavoschi et al., 2020). Following this trend of research, we aim to investigate whether Twitter data can reveal people's information privacy concerns. Thus, our first research question is as follows:

- RQ1: Which information privacy concerns are present over sccial media content about a data-breach scandal?

As we have reviewed in the prior section, there are arguments and evidence to support that information privacy concerns can vary across culture, language, and demographics (Chai, 2020; Li et al., 2020; Oghazi et al., 2020; Oghazi et al., 2020; González et al., 2019a). If information privacy concerns are present in a Twitter dataset, we could explore how they differ across people who live in different parts of the world and those who speak different languages. As we do not expect any specific trend of differences, we propose to test the following null hypotheses:

- H0a. There are no differences in information privacy concerns by language

- H0b. There are no differences in information privacy concerns by world region.

\section{Data \& Methods}

To answer our research question and test the hypotheses, we implemented a four-step methodology (see Fig 1). We retrieved tweets associated with data privacy during a specific period (4.1. data collection). We filtered the data, removing retweets and excluding tweets likely to be generated by bots (4.2. data pre-processing). We created word-embeddings (a multi-dimensional representation of a corpus) for the remaining tweets according to their language and world region (4.3. text mining). Finally, we conducted an analysis to identify similarities and differences in the semantic contexts of privacy keywords in the word embeddings (4.4. coding and analysis). Details about each of these steps are presented below.

\subsection{Data Collection}

We retrieved tweets related to the Facebook and Cambridge Analytica scandal between April 1st and July 10th, 2018. We focused on tweets in Spanish and English.

On March 17, 2018, it was revealed that the data firm Cambridge Analytica used personal data of 87 million Facebook users for political advertising purposes without their consent (Schneble et al., 2018; Oghazi et al., 2020). This scandal caused 


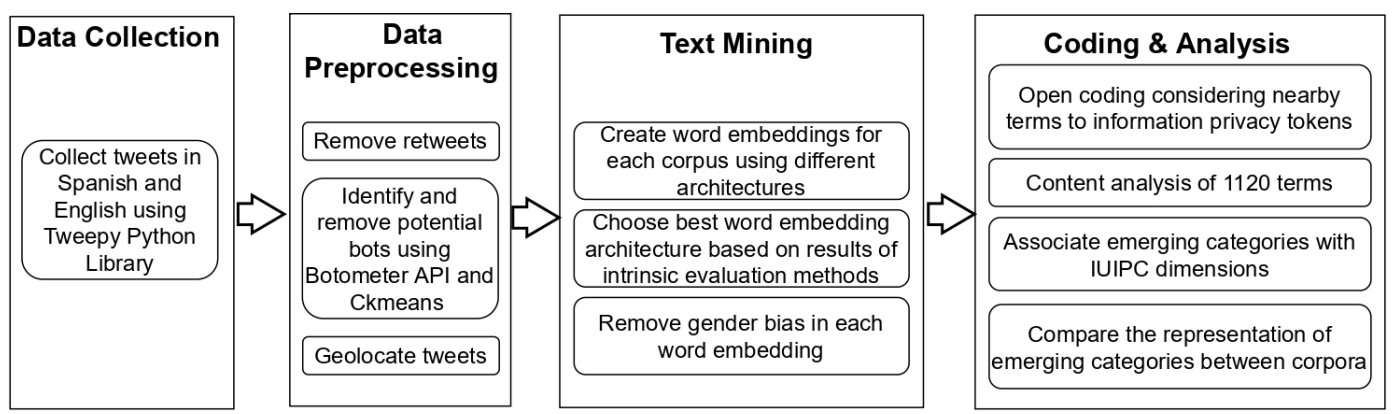

Figure 1. Methodology flow chart

the closure of Cambridge Analytica (Solon and Laughland, 2018) and numerous lawsuits against Facebook in the USA and the European Union. On Twitter, a \#DeleteFacebook campaign started as a response to this scandal (Lin, 2018). As the Cambridge Analytica scandal triggered Twitter users from different world regions (who speak diverse languages) to spontaneously share their opinions, experiences, and perspectives about data privacy, we decided to use a sample of these tweets to answer our research question and test our hypotheses.

We used Tweepy ${ }^{1}$ to collect relevant tweets. Tweepy is a Python library for accessing the standard real-time streaming Twitter API, ${ }^{2}$ which allows to freely retrieve tweets that match a given query. If the query is too broad that it includes over $1 \%$ of the total number of tweets posted at that time worldwide, the query's response is sampled (Aghababaei and Makrehchi, 2017; Morstatter et al., 2014). The way in which Twitter samples the data is unpublished. Nevertheless, studies have shown that as more data from the API is retrieved, a more representative sample of the Twitter stream is obtained (Leetaru, 2019; Morstatter et al., 2013).

To obtain relevant tweets, we used Tweepy's language filter to retrieve tweets in Spanish or English. We manually crafted a list of hashtags and keywords related to the Cambridge Analytica scandal. We collected tweets that had at least one of these terms. Examples of these terms are: "\#DeleteFacebook", "\#CambridgeAnalytica", "\#Mark Zuckerberg", "Facebook", "Facebook Cambridge", and "Facebook data breach". Additionally, when appropriate, we added translations to Spanish of these terms to build the Spanish dataset. ${ }^{3}$ In this way, if a tweet in Spanish had a hashtag in English, the tweet was collected and added to the Spanish dataset. A full list of the terms used to retrieve our data is available online ${ }^{4}$.

Following this procedure, we retrieved more than 470,000 tweets in Spanish and more than 7.4 million tweets written in English (see Table II). The tweets in Spanish were produced by approximately 220,000 users while tweets in English were generated by about 1.8 million unique Twitter accounts.

\footnotetext{
${ }^{1}$ http://www.tweepy.org/

${ }^{2}$ https://developer.twitter.com/en/docs/tweets/filter-realtime/guides/basic-streamparameters.html

3 The authors are fairly confident of the quality of these translations because some of them are Spanish native speakers while others are English native speakers

${ }^{4}$ https://github.com/gonzalezf/Regional-Differences-on-Information-Privacy-Concerns
} 
Table II. Datasets before and after data cleaning

\begin{tabular}{lrrrrr}
\hline Dataset & \multicolumn{2}{c}{ Spanish } & & \multicolumn{2}{c}{ English } \\
\cline { 2 - 3 } \cline { 6 - 6 } & \#Tweets & \#Accounts & & \#Tweets & \#Accounts \\
\hline Total & 472,363 & 222,352 & & $7,476,988$ & $1,846,542$ \\
Original & 106,656 & 47,951 & & $1,572,371$ & 574,452 \\
With Botometer score & 100,606 & 44,182 & & $1,442,112$ & 504,214 \\
Human-owned & 74,644 & 36,056 & & 975,678 & 410,180 \\
\hline
\end{tabular}

\subsection{DATA PRE-PROCESSING}

As we meant to analyze people's opinions about information privacy, we decided to pre-process our data in three ways. We removed all retweets to avoid analyzing exact duplicates. Afterwards, we sought to identify and filter out tweets that were generated by bots. Our last step was to associate tweets with different world regions. We further explain each of these steps below.

First, we excluded retweets to avoid analyzing exact duplicates of content. This methodology step is suggested by several authors (Hajjem and Latiri, 2017; AgüeroTorales et al., 2021; Singh et al., 2020). We kept tweets, quoted tweets, and replies to tweets. Exclusion of retweets reduced our datasets' size by $80 \%$. We refer to the resulting datasets as original tweets (see Table II).

We used Botometer (Davis et al., 2016) to detect and remove tweets created by bots. Botometer uses machine-learning to analyse more than one thousand features (Badawy et al., 2018) including tweets' content and sentiment, accounts' and friends' metadata, retweet/mention network structure, and time series of activity (Varol et al., 2017; Yang et al., 2019) to generate a score that ranges from 0 to 1. A higher value suggests a high likelihood that an inspected account is a bot (Badawy et al., 2018). This tool has reached high accuracy (94\%) in predicting both simple and sophisticated bots (Varol et al., 2017; Badawy et al., 2018). Botometer is free and has been widely used ${ }^{5}$ (Varol et al., 2017; Yang et al., 2019).

Botometer processed all of the Twitter accounts who wrote original tweets. It returned a score for 44,182 $(92.14 \%)$ and $504,214(87.77 \%)$ accounts of the Spanish and English datasets, respectively. Botometer cannot generate scores for suspended accounts or those that have their tweets protected. We decided to remove the tweets from these accounts from our datasets because we cannot confidently claim that they come from humans' accounts. We applied the Ckmeans (Wang and Song, 2011) algorithm to define a threshold to distinguish between humans' and bots' accounts. For each language, we clustered the Botometer scores into five groups, where the first group included the accounts with the lowest scores (more human-like) and the fifth group comprised those with the highest scores (more bot-like). After manually inspecting the accounts around the thresholds of each group, we concluded that the fourth and fifth groups in each dataset were unlikely to contain human accounts. Therefore, we used the fourth group's lowest threshold to discriminate humans' and bots' accounts. Accounts with a score lower than 0.4745 and 0.4947 in Spanish and English, respectively, were considered as human-owned. These thresholds are similar

\footnotetext{
${ }^{5}$ Since its release in May 2014, Botometer has served over one million requests (Davis et al., 2016) via its website (https://botometer.iuni.iu.edu) and its Python API (https://github.com/IUNetSci/botometer-python)
} 
to those used in related work, where scores lower than 0.5 had been considered as humans (Varol et al., 2017; Badawy et al., 2018). As a result, our datasets contain 36,056 human-owned accounts that created 74,644 tweets in Spanish and 410,180 accounts that created 975,678 tweets in English.

Finally, we used the GeoNames $\mathrm{API}^{6}$ to identify the country of residence of Twitter users in our datasets. On Twitter, users can self-report their city or country of precedence. Nevertheless, textual references to geographic locations can be ambiguous. For example, over 60 different places around the world are named "Paris" (Jackoway et al., 2011). To deal with this challenge, we employed the GeoNames API, which is a collaborative gazetteer project that contains more than 11 million entries and alternate names for locations around the world in a variety of different languages (Bergsma et al., 2013). Given a text, its algorithm performs operations to recognize potential locations, followed by a disambiguation process. This last step checks hierarchical relations and picks a location by their proximity to other locations mentioned in the text (Lagos, 2017). This tool has yielded results with an accuracy above $80 \%$ (Jackoway et al., 2011).

We found that $80.68 \%$ of users in our Spanish dataset and $78.68 \%$ of users in our English dataset had filled the city or country fields in their profiles. However, the GeoNames API could not detect the users' location in several cases, for example when inaccurate information was provided (e.g., "Planet Earth.. where everyone else is from", "Mars"). Nonetheless, the tool was able to identify the location of users who created $58.7 \%$ of the Spanish tweets and $59.9 \%$ of the English ones. In the Spanish dataset, most tweets came from Spain (16.5\%) and Latin American countries, such as Mexico (11.9\%), Argentina (6.2\%), and Venezuela (4.7\%). In the English dataset, the majority of tweets came from the United States $(32.4 \%)$, followed by United Kingdom (6.9\%), India (3.2\%), and Canada (2.7\%) (see Table III).

Table III. Top-10 most frequent user locations in the Spanish and English datasets

\begin{tabular}{|c|c|c|c|c|c|c|c|c|c|}
\hline \multicolumn{5}{|c|}{ Spanish } & \multicolumn{5}{|c|}{ English } \\
\hline \multirow[b]{2}{*}{ Location } & \multicolumn{2}{|c|}{ Tweets } & \multicolumn{2}{|c|}{ Users } & \multirow[b]{2}{*}{ Location } & \multicolumn{2}{|c|}{ Tweets } & \multicolumn{2}{|c|}{ Users } \\
\hline & \# & $\%$ & \# & $\%$ & & $\#$ & $\%$ & \# & $\%$ \\
\hline Spain & 12,342 & 16.5 & 5,483 & 15.2 & U.S & 315,913 & 32.4 & 132,155 & 32.2 \\
\hline Mexico & 8,852 & 11.9 & 4,720 & 13.1 & U.K & 66,901 & 6.9 & 29,656 & 7.2 \\
\hline Argentina & 4,648 & 6.2 & 2,505 & 6.9 & India & 30,781 & 3.2 & 12,424 & 3.0 \\
\hline Venezuela & 3,518 & 4.7 & 1,094 & 3.0 & Canada & 26,487 & 2.7 & 11,564 & 2.8 \\
\hline Colombia & 2,447 & 3.3 & 1,348 & 3.7 & Australia & 13,375 & 1.4 & 6,501 & 1.6 \\
\hline U.S & 1,823 & 2.4 & 948 & 2.6 & Germany & 9,260 & 0.9 & 3,493 & 0.9 \\
\hline Chile & 1,806 & 2.4 & 1,073 & 3.0 & France & 8,605 & 0.9 & 3,006 & 0.7 \\
\hline Peru & 1,116 & 1.5 & 619 & 1.7 & Nigeria & 5,156 & 0.5 & 2,787 & 0.7 \\
\hline Ecuador & 893 & 1.2 & 455 & 1.3 & U.A.E & 5,120 & 0.5 & 1,504 & 0.4 \\
\hline Brazil & 587 & 0.8 & 172 & 0.5 & South Africa & 4,962 & 0.5 & 2,912 & 0.7 \\
\hline Other & 5,815 & 7.8 & 3,100 & 8.6 & Other & 98,100 & 10.1 & 42,658 & 10.4 \\
\hline Unknown & 30,797 & 41.3 & 14,574 & 40.4 & Unknown & 391,018 & 40.1 & 161,767 & 39.4 \\
\hline
\end{tabular}

To compare information privacy concerns by geographical regions, we divided the Spanish Twitter dataset in two sets: tweets written by users from (1) Latin America

${ }^{6}$ http://www.geonames.org/ 
and (2) Europe. Similarly, we categorized the English dataset into three groups: tweets written by users from (1) North America, (2) Europe and (3) Asia. As a result, five different language-regional datasets were generated. The Spanish-Latin America dataset includes 27,839 tweets written by 13,937 users. The Spanish-Europe dataset comprises 12,799 tweets created by 5,774 accounts. Regarding the English data, the North America dataset includes 342,400 tweets generated by 142,719 users, the English-Europe one has 111,745 tweets of 46,927 users, and the English-Asia dataset contains 42,208 tweets produced by 17,929 accounts (Table IV). We did not consider other subsets because of their small size. In Spanish, we only collected 1,929 tweets from North America and 217 tweets from Asia. In English, we only collected 3,851 tweets from Latin America.

Table IV. Tweets and users in each dataset

\begin{tabular}{llrr}
\hline Language & Region & \# of tweets & \# of users \\
\hline \multirow{2}{*}{ Spanish } & Latin America & 27,839 & 13,937 \\
& Europe & 12,799 & 5,774 \\
\hline \multirow{3}{*}{ English } & North America & 342,400 & 143,719 \\
& Europe & 111,745 & 46,927 \\
& Asia & 42,208 & 17,929 \\
\hline
\end{tabular}

\subsection{TeXt mining: Word embeddings to identify Semantic COnteXts}

We employed word embeddings (Mikolov et al., 2013) to characterize the semantic context in which privacy-related keywords are framed. Based on co-occurrence of terms, word embeddings create a reduced multi-dimensional representation of a corpus. Such representation can be used to analyze the semantic proximity among the corpus' terms. Analyzing the closest terms of a given term can reveal the semantic context in which it is used (Rho et al., 2018; González et al., 2019a).

We created a set of word embeddings to enable cross-language and cross-regional comparisons. First, we built word embeddings for the Spanish and English datasets (containing both geolocated and non-geolocated tweets). Then, we generated word embeddings for each of our five language-regional datasets. Before creating the word embeddings, we transformed the text to lowercase. We also removed stop-words and digits from the tweets. We customized our stop-words to ensure that symbols like "\#" were removed but not the words that follow it. Links and usernames were removed. Words with total frequency lower than three were ignored. These steps downsized the vocabulary by approximately $67 \%$ (details in Table V).

We considered eight word embedding architecture combinations that involve Word2Vec/FastText, CBOW/Skipgram and different numbers of dimensions and epochs. As there is still no consensus about which word embedding evaluation method is more adequate (Wang et al., 2019), we evaluated each word embedding architecture for the English dataset over 18 intrinsic conscious evaluation methods (Wang et al., 2019) using a word embedding benchmark library. ${ }^{7}$ Wang et al. (2019) approach has categorized the evaluation methods in three categories:

\footnotetext{
${ }^{7}$ https://github.com/kudkudak/word-embeddings-benchmarks
} 
Table V. Initial and final vocabulary size in each dataset

\begin{tabular}{rlrr}
\hline Language & Region & Initial vocabulary size & Final vocabulary size \\
\hline \multirow{3}{*}{ Spanish } & All & 65,036 & 21,736 \\
\cline { 2 - 4 } & Latin America & 35,149 & 11,359 \\
& Europe & 21,630 & 6,696 \\
\hline \multirow{5}{*}{ English } & All & 244,371 & 76,128 \\
\cline { 2 - 4 } & North America & 115,710 & 41,109 \\
& Europe & 66,042 & 23,514 \\
& Asia & 39,120 & 13,896 \\
\hline
\end{tabular}

- Word semantic similarity (WSS): RW, MEN, Mturk287, WS353R, WS353S, WS353, SimLex999, RG65 and TR9856

- Word Analogy (WA): Google Analogy Test set, MSR and SemEval 2012-2

- Concept categorization (CC): AP, BLESS, BM, ESSLI 1A, ESSLI 2B, and ESSLI 2C

To choose the best architecture, we designed a point system to reflect the embeddings' performance. For each evaluation method, the word embedding with the highest accuracy received a score of 8 points, the embedding with the second highest accuracy was assigned 7 points, and so on. After running all evaluation methods, we summed the points obtained for each architecture. Considering a negative sampling and windows size parameters equal to 5, a Word2Vec CBOW architecture with 300 dimensions trained during 50 epochs achieved the total highest score (see Table VI). The same architecture had the best performance for all English regional datasets. Given that these evaluation methods are not available for a Spanish corpus, the same architecture was used to create all the Spanish word embeddings.

Table VI. Word embedding architectures and their evaluation scores. Best performance is indicated with bold font style.

\begin{tabular}{|c|c|c|c|c|c|c|c|}
\hline \multicolumn{4}{|c|}{ Architecture } & \multicolumn{4}{|c|}{ Evaluation word embedding score } \\
\hline Type & Model & Dim. & Epochs & WSS & WA & $\mathrm{CC}$ & Total \\
\hline FastText & CBOW & 100 & 10 & 22 & 19 & 25 & 66 \\
\hline Word2Vec & Skipgram & 100 & 10 & 40 & 4 & 35 & 79 \\
\hline Word2Vec & CBOW & 100 & 10 & 35 & 11 & 33 & 79 \\
\hline Word2Vec & CBOW & 100 & 50 & 34 & 16 & 41 & 91 \\
\hline Word2Vec & CBOW & 100 & 300 & 33 & 9 & 31 & 73 \\
\hline Word2Vec & CBOW & 300 & 10 & 40 & 18 & 32 & 90 \\
\hline Word2Vec & CBOW & 300 & 50 & 53 & 21 & 36 & 110 \\
\hline Word2Vec & CBOW & 300 & 300 & 31 & 10 & 29 & 70 \\
\hline
\end{tabular}

Previous work has reported that word embeddings can reflect gender bias as a result of social constructs embedded in the data (Zhao et al., 2018; Jha and Mamidi, 2017). To reduce gender bias while preserving its useful properties such as the ability 
to cluster related concepts, we followed Bolukbasi et al. (2016) approach. This is a post-processing method that projects gender-neutral words to a subspace which is perpendicular to a gender dimension, defined by a set of terms associated with gender such as girl, boy, mother and father (Zhao et al., 2018). We applied the following procedure to our English embeddings: (1) we identified a gender subspace selecting pairs of English words that can reflect a gender direction in each word embedding such as woman-man, daughter-son and female-male, (2) we ensured that gender neutral words are zero in the gender subspace, and (3) we made neutral words equidistant to all pair of terms contained in a collection of equality sets. A equality set is composed by a pair of words that should differ only in the gender component such as $\{$ grandmother, grandfather $\}$ and $\{$ guy, gal $\}$. During this process, we used the English terms suggested by Bolukbasi et al. (2016). For the Spanish word embeddings, we used Google Translate $\mathrm{API}^{8}$ to translate the same terms.

\subsection{Manual CODing \& ANALYSis}

We conducted a systematic qualitative examination of the semantic contexts in which information privacy terms appear according to the word embeddings. First, we conducted open coding of the semantic neighborhoods of privacy-related keywords. After several iterations, we developed a set of categories to characterize them. To assess if information privacy concerns were present (RQ1), we contrasted these categories to a widely accepted framework to describe internet users' information privacy concerns.

We focused our investigation on four keywords in English: information, privacy, users and company. We used their corresponding translations in Spanish: información, privacidad, usuarios and empresa. We chose to include information and privacy because they are the main concepts under study. We could have added data; however, its semantic context is almost identical to that of information. Thus, adding it would have resulted in a mere duplication of terms. To increase the size of our dataset, we decided to add users and company because of their key roles in respect of controlling and safeguarding personal information. We also considered these terms more specific to the vocabulary of the data privacy domain than alternative ones (e.g., people, organizations).

For each embedding, we retrieved the closest terms to the four keywords. Closeness between each term and a keyword was measured using cosine similarity. For instance, the closest terms retrieved to the keyword information in the English word embedding were info, data, details, and personal, in that order. We chose to study the 40 closest terms after careful examination of the lists of close terms according to our different embeddings. After the 40th position in these lists, we rarely found terms that were even slightly related to information privacy. We reason that the value of this threshold is dataset-dependent. It is likely to be related to the vocabulary sizes (ours range from 6,696 to 41,109). In our case, we opted for using 40 as the threshold to study the semantic context of each keyword. Hence, we qualitatively analyzed 160 terms for each embedding. Overall, our dataset for qualitative analysis included 1,120 terms.

Two of the authors conducted open coding of the 320 terms retrieved from the Spanish and English word embeddings. Open coding is a process to identify, define and develop categories based on properties and dimensions of raw data (Williams and Moser, 2019). We used this technique to identify distinct concepts and themes from

8 https://cloud.google.com/translate/ 
the extracted terms (Williams and Moser, 2019). After inspecting the retrieved terms during several iterations, the coders developed a coding guideline with multiple concept categories and their corresponding explanations to classify the retrieved terms. For example, the term info extracted from the keyword information was categorized as a synonymous, given that we can attribute to it the same meaning. The terms data and details were classified as data \& information, and personal was labeled as attribute or characteristic. During a series of meetings, both coders compared their categorization process and refined a common coding guideline, establishing rules that would increase the categorization's reliability. The goal during this process is to segregate, group, regroup and re-link the terms to consolidate meaning and explanation of the categories (Williams and Moser, 2019).

At the end of this process, 15 categories emerged from the data (see Table X). Considering the four keywords, an inter-coder reliability measure (Cohen's kappa) of 0.685 and 0.754 were obtained for the Spanish and English dataset, respectively. These scores indicate substantial agreement (Viera et al., 2005) during the process.

We repeated the procedure for the regional datasets. The coders categorized the 40 closest terms to the keywords according to the coding guideline. Through an iterative process, a total of 800 words were manually coded. No new categories emerged from the data. On average, a Cohen's kappa above 0.722 was obtained in all the regional datasets.

Table VII. Inter-rater reliability (Cohen's kappa) score by dataset

\begin{tabular}{llrrrrr}
\hline Language & Region & Information & Privacy & Company & Users & Avg. by dataset \\
\hline \multirow{3}{*}{ Spanish } & All & 0.864 & 0.630 & 0.604 & 0.642 & $\mathbf{0 . 6 8 5}$ \\
& Latin America & 0.749 & 0.673 & 0.687 & 0.778 & $\mathbf{0 . 7 2 2}$ \\
& Europe & 0.820 & 0.710 & 0.774 & 0.827 & $\mathbf{0 . 7 8 3}$ \\
\hline \multirow{2}{*}{ English } & All & 0.768 & 0.747 & 0.672 & 0.829 & $\mathbf{0 . 7 5 4}$ \\
& North America & 0.831 & 0.721 & 0.912 & 0.971 & $\mathbf{0 . 8 5 9}$ \\
& Europe & 0.777 & 0.743 & 0.805 & 0.807 & $\mathbf{0 . 7 8 3}$ \\
\hline \multirow{2}{*}{ Average of all embeddings } & 0.832 & 0.685 & 0.736 & 0.833 & $\mathbf{0 . 7 7 1}$ \\
\hline
\end{tabular}

To assess if information privacy concerns were present in a Twitter dataset about a data-breach scandal (RQ1), we compared the resulting categories with the IUIPC's dimensions: collection, control, and awareness. IUIPC is a theory-based model that has been widely used to study information privacy concerns on the internet (see Section 2.1.2). Then, we tested the null hypotheses about differences in information privacy concerns across language and world regions (H0a and HOb). To do so, we used a Chi-squared test to assess if the proportion of terms in the semantic contexts were significantly different across word embeddings. In all of these tests, we accounted for multiple comparisons by applying alpha adjustment according to Šidák (Šidák, 1967; Haynes, 2013). This method allowed us to control the probability of making false discoveries when performing multiple hypotheses tests. 


\section{Results}

In this section, we address our research question and test the null hypotheses about differences in information privacy concerns by language and world regions.

As explained above, we create word embeddings for our Spanish and English datasets of tweets about the Cambridge Analytica scandal. Then, we take a closer examination of how the semantic context of four keywords varies across language and world regions. The semantic context is operationalized as the 40 closest terms of each keyword: information, privacy, company, and users. As an example, Table VIII and Table IX show the 20 closest terms to the keywords, according to the Spanish and English word embeddings. ${ }^{9}$ Full results are available online. ${ }^{10}$

Table VIII. Top 20 closest terms to information and privacy in the Spanish and English word embeddings

\begin{tabular}{lllll}
\hline \multicolumn{2}{c}{ Information } & & \multicolumn{2}{c}{ Privacy } \\
\cline { 1 - 2 } Spanish & English & & Spanish & English \\
\hline data & info & intimacy & data privacy \\
third parties & data & & data & gdpr \\
fact & personal & & confidentiality & protection \\
third & users & & personal data & users \\
interviewer & profiles & & privacy policy & user \\
facebook & identifiers & & digital security & consumers \\
users & personal data & & data protection & data \\
privacy & private & & identity & transparency \\
consent & records & & minor & facebook \\
authorization & user & & facebook & personal \\
purposes & consent & & third parties & consent \\
private & advertisers & information & security \\
personal & permission & cibersecurity & sharing \\
location & metadata & sensitive & data protection \\
ecomlancer & datas & emails & tos \\
serve & companies & cookies & consumer \\
viatec & individuals & protect yourself & collection \\
intimate & freely & suppose & opt \\
profiles & informations & take care of your data & privacyrights \\
\hline & & & & trust \\
\hline
\end{tabular}

\subsection{INFORMATION PRIVACY CONCERNS PRESENT IN A TWITTER DATASET}

As a result of the coding process, we define 15 categories to analyze the closest terms (see Table X). To answer our first research question, we compare our categories with IUIPC, a framework widely used to measure information privacy concerns in the context of the Internet (Liu and Carter, 2018). We find relationships among some of our categories and the three IUIPC concepts as well as our initial keywords, as shown in Figure 2.

\footnotetext{
${ }^{9}$ Terms in Spanish were translated to English by the authors

10 https://github.com/gonzalezf/Regional-Differences-on-Information-Privacy-Concerns
} 
Table IX. Top 20 closest terms to 'company and users in the Spanish and English word embeddings

\begin{tabular}{|c|c|c|c|}
\hline \multicolumn{2}{|c|}{ Company } & \multicolumn{2}{|l|}{ Users } \\
\hline Spanish & English & Spanish & English \\
\hline company & companies & third parties & user \\
\hline consultant & firm & sensitive & consumers \\
\hline firm & companys & citizens & personal \\
\hline organization & platform & illegally & peoples \\
\hline obtain & firms & users & subscribers \\
\hline relation & organization & authorization & customers \\
\hline researcher & data & profiles & people \\
\hline deliver & entity & private & facebook \\
\hline plot & giant & used & data \\
\hline finance & user & people & $\mathrm{fb}$ \\
\hline ltd & facebook & illegal & apps \\
\hline way & corporation & clients & individuals \\
\hline facebook & $\mathrm{fb}$ & user & advertisers \\
\hline illegally & organisation & improperly & privacy \\
\hline companies & business & obtained & information \\
\hline $\mathrm{ca}$ & users & nametests & app \\
\hline own & businesses & information & citizens \\
\hline brand & service & facebook & profiles \\
\hline decide & $\mathrm{ca}$ & data & companies \\
\hline $\mathrm{scl}$ & site & voters & collected \\
\hline creole & personal & cambridgeanalytics & private \\
\hline relations & organizations & infringement & consent \\
\hline cambridge & employees & use & accounts \\
\hline data & agency & purposes & permissions \\
\hline laboratories & $\mathrm{co}$ & authorized & use \\
\hline
\end{tabular}

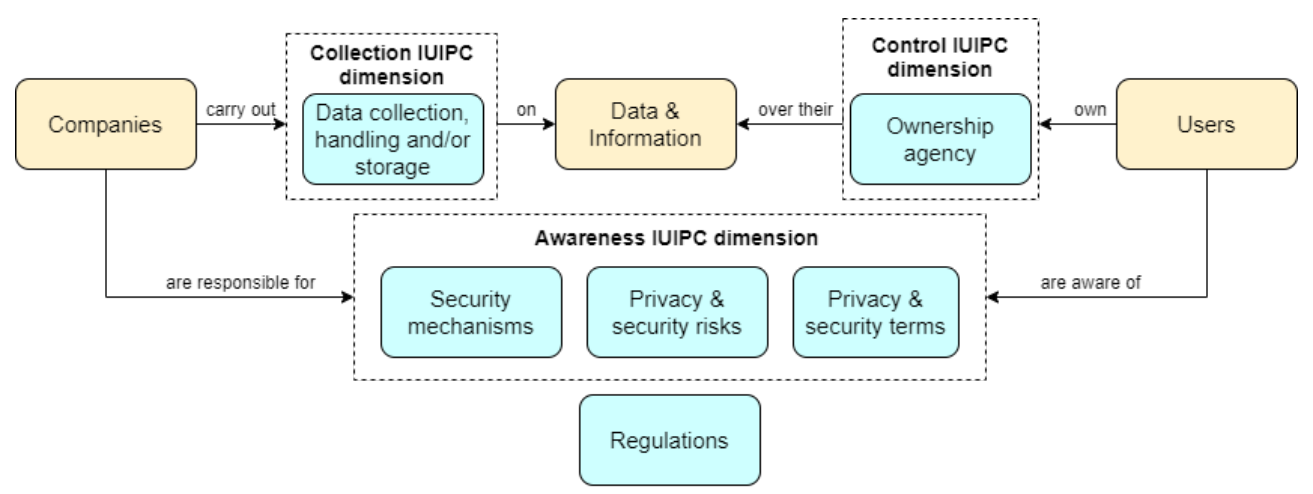

Figure 2. Relationships between our categories and IUIPC dimensions

Three categories match our initial keywords (Table X, yellow background): (1) data \& information is associated with the information keyword, including direct references to this concept and examples of user data and its meaning (e.g., "messages" and "metadata"), (2) companies include terms about organizations that use per- 
Table X. Coding guideline to classify the semantic contexts of our keywords. Yellow background is for categories that match our initial keywords. Light blue background is for categories related to IUIPC. Gray background is for other categories.

\begin{tabular}{|c|c|c|c|}
\hline Categories & Description & Spanish Examples & English Examples \\
\hline $\begin{array}{l}\text { Data \& Informa- } \\
\text { tion }\end{array}$ & $\begin{array}{l}\text { Direct references to these } \\
\text { concepts and examples of } \\
\text { user data and its meaning }\end{array}$ & $\begin{array}{l}\text { records, location, data, } \\
\text { emails, profiles, contacts }\end{array}$ & $\begin{array}{l}\text { profile, messages, documents, } \\
\text { location, accounts, metadata }\end{array}$ \\
\hline Companies & $\begin{array}{l}\text { Entities that manipulate } \\
\text { user data for their own pur- } \\
\text { poses }\end{array}$ & $\begin{array}{l}\text { facebook, cambridgeanalyt- } \\
\text { ica, scl, grindr, advertisers }\end{array}$ & $\begin{array}{l}\text { facebook, cambridgeanalyt- } \\
\text { ica, google, emerdata, apple }\end{array}$ \\
\hline Users & Data owners & $\begin{array}{l}\text { users, consumers, popula- } \\
\text { tion, citizens, people }\end{array}$ & $\begin{array}{l}\text { users, consumers, } \\
\text { subscribers, citizens, people }\end{array}$ \\
\hline $\begin{array}{l}\text { Data collection, } \\
\text { handling and/or } \\
\text { storage }\end{array}$ & $\begin{array}{l}\text { Mechanisms and verbs asso- } \\
\text { ciated with obtaining, col- } \\
\text { lecting and handling data }\end{array}$ & $\begin{array}{l}\text { use, obtained, log in, collect- } \\
\text { ing, apps, mechanisms }\end{array}$ & $\begin{array}{l}\text { collected, store, access, } \\
\text { databases, usage, analyzed }\end{array}$ \\
\hline $\begin{array}{l}\text { Ownership } \\
\text { agency }\end{array}$ & $\begin{array}{l}\text { User-control over personal } \\
\text { information }\end{array}$ & $\begin{array}{l}\text { cization, agree, consent, } \\
\text { t }\end{array}$ & $\begin{array}{l}\text { consent, opt, shield, auton- } \\
\text { omy }\end{array}$ \\
\hline $\begin{array}{l}\text { Privacy \& secu- } \\
\text { rity terms }\end{array}$ & $\begin{array}{l}\text { ssociated with data } \\
\text { and security }\end{array}$ & $\begin{array}{l}\text { entiality, } \\
\text { e }\end{array}$ & $\begin{array}{l}\text { confidenciality, transparency, } \\
\text { privately, dataprivacy, secu- } \\
\text { rity }\end{array}$ \\
\hline $\begin{array}{l}\text { Security mecha- } \\
\text { nisms }\end{array}$ & $\begin{array}{l}\text { Tools and tec } \\
\text { implement secu }\end{array}$ & d, biome- & $\begin{array}{l}\text { encrypted, password, biomet- } \\
\text { ric, privacybydesign }\end{array}$ \\
\hline $\begin{array}{l}\text { Privacy \& secu- } \\
\text { rity risks }\end{array}$ & $\begin{array}{l}\text { Entities or bad practices } \\
\text { that can compromise sensi- } \\
\text { tive data }\end{array}$ & $\begin{array}{l}\text { trojan, cybercriminal, ille- } \\
\text { gally, scams, stealing }\end{array}$ & $\begin{array}{l}\text { grooming, da } \\
\text { use, illegally, }\end{array}$ \\
\hline Regulation & $\begin{array}{l}\text { Law, rule or regulatic } \\
\text { controls the use of use }\end{array}$ & $\begin{array}{l}\text { rgpd, right to be forgotten, } \\
\text { privacypolicy, iso, habeas- } \\
\text { data }\end{array}$ & $\begin{array}{l}\text { gdpr, tos, hippa, } \\
\text { privacyrights, regulation }\end{array}$ \\
\hline tymous & $\begin{array}{l}\text { Same meaning than the key- } \\
\text { word }\end{array}$ & $\begin{array}{l}\text { info, company, firm, user, pri- } \\
\text { vate }\end{array}$ & $\begin{array}{l}\text { info, companies, firm, pri- } \\
\text { vacy, user, }\end{array}$ \\
\hline $\begin{array}{l}\text { Attribute or } \\
\text { characteristic }\end{array}$ & $\begin{array}{l}\text { A characteristic of the key- } \\
\text { word }\end{array}$ & $\begin{array}{l}\text { false, private, external, spe- } \\
\text { cialized, britain }\end{array}$ & $\begin{array}{l}\text { sensitive, giant, holistic, } \\
\text { strategic, affected }\end{array}$ \\
\hline Action & $\begin{array}{l}\text { Action or activity linked to } \\
\text { the keyword }\end{array}$ & $\begin{array}{l}\text { define, explode, promote, at- } \\
\text { tend, move }\end{array}$ & $\begin{array}{l}\text { order, solve, reveal, update, } \\
\text { confirming }\end{array}$ \\
\hline Third party & $\begin{array}{l}\text { Can not be categorized as } \\
\text { User or Company. There is } \\
\text { not sufficient contextual in- } \\
\text { formation to do so }\end{array}$ & $\begin{array}{l}\text { rrhh, medicians, interviewer, } \\
\text { ex employee, philippine }\end{array}$ & $\begin{array}{l}\text { government, agency, indians, } \\
\text { europeans, developers, }\end{array}$ \\
\hline $\begin{array}{l}\text { Reaction or atti- } \\
\text { tude }\end{array}$ & $\begin{array}{l}\text { Way of feeling or acting to- } \\
\text { ward a entity }\end{array}$ & $\begin{array}{l}\text { guilt, honest, overfall, suffers, } \\
\text { handle }\end{array}$ & $\begin{array}{l}\text { willingly, freely, tighter, re- } \\
\text { stricting, forced }\end{array}$ \\
\hline Undetermined & $\begin{array}{l}\text { Relationship between key- } \\
\text { word and term is unknown }\end{array}$ & $\begin{array}{l}\text { approximately, v.i.p, ground, } \\
\text { higher, depth }\end{array}$ & $\begin{array}{l}\text { psychological, millions, new, } \\
\text { group, image }\end{array}$ \\
\hline
\end{tabular}

sonal data for their own purposes such as "Facebook" and "Apple", and (3) users contain references to this keyword (e.g., "customers", "people").

Five categories are related to IUIPC (Table X, light blue background). We identify a data collection, handling and/or storage category that contains words associated with technology or techniques useful to obtain, collect or handle data (e.g: "databases", "services", "app", "website"). This matches to the IUIPC's collection dimension, which refers to the "degree to which a person is concerned about the amount of individual-specific data possessed by others relative to the value of benefits received"(Malhotra et al., 2004). Examples of tweets that include terms that fit this category are:

\footnotetext{
'@hidden_username @hidden_username This is bigger than facebook because all social media outlets collect and store this data on every user. If you haven't looked,
} 
check and see what twitter has collected on you. Free apps are not free, neither are paid ones'

'Facebook collects and sells PII data. Google and others maintain behavioral data anonymously and serve ads against it, but don't connect that data to identities that are sold to advertisers. I was not aware Facebook was such an anomaly.'

The IUIPC's control dimension denotes concerns about control over personal information. This is often exercised through approval, modification and opportunity to opt-in or opt-out (Malhotra et al., 2004). Terms related to this dimension appeared in the coding phase (e.g., "consent", "opt", "permission") and were categorized as ownership agency. This category also includes advice directed to users and good privacy practices terms (e.g., "prevent", "protect"). Examples of tweets related to this category are:

'If anything we should learn from the \#Facebook data breach. Don't volunteer information and prevent that secondary data collection by using \#adblocker and \#VPN'

'Cambridge Analytica whistleblower Christopher Wylie urges U.S. senators to focus less on data consent and more on the idea that it's almost impossible to opt out of, for example, Google.'

The third IUIPC's dimension is awareness, which refers to individual concerns about her/his awareness of organizational information privacy practices (Malhotra et al., 2004). Three of our categories are associated with this dimension: (1) privacy and security terms that include words associated with data privacy and security such as "confidentiality", "transparency" and "safety"; (2) security mechanisms that refers to tools and techniques that implement security services (e.g., "password", "encryption"); and, (3) privacy \& security risks that denote entities or bad practices that can compromise sensitive data, for example: "troyano" (trojan), "databreach", "grooming" and "ciberdelincuente" (cybercriminal). Tweets that use these terms are:

'Hmm- what do you think? I forsee a wave of new social network startups- will any be able to rise? Besides privacy and transparency what else would you want from a social network? \#swtech'

'WhatsApp Co-Founder To Leave Company Amid Disagreements With Facebook. Facebook's desire to weaken WhatsApp's encryption and collect more personal data reportedly fueled the decision'

'Canadian federal privacy officials warned that third-party developers' access to Facebook users' personal information raises serious privacy risks back in 2009. @hidden_link'

Another privacy-related category emerges from our coding but can not be easily associated with an IUIPC dimension. This is the regulation category, which includes terms associated with laws and rules that control the use of personal data such as "gdpr" in reference to the European General Data Protection Regulation or "tos" in reference to Terms of Services. Examples of tweets with these terms are:

'New regulation in Europe called gdpr makes companies liable for data breaches with penalties which include fines of a percentage of global turnover. It feels like all Zuckerberg is liable for is a slap on the wrist and having to apologise in public' 
'\#Today we are confirming that multiple snippets of data from $\mathrm{Cl}$ that was lifted from facebook are in Russia. If you are an EU citizen this means you have a right to sue both companies for gdpr based infringements. We will be leading this cause should no one else step up....'

'Senator to \#Zuckerberg: Your terms of services are only a few pages long. People complain when online contracts are too long and filled with legalese. Now lawmakers are complaining they're too short. What's the threshold for length and detail, and how do we decide?'

Other categories are identified as well (Table X, gray background). The attribute or characteristic category contains modifiers of a specific keyword. For example, the term "sensitive" emerges from the closest terms to information, and the term "britanica" (British) appears among the nearest terms to company. The action category includes words related to an act. For instance, the verbs "obtener" (obtain) and "entregar" (deliver) come out among the closest terms to company. The third party category contains terms related to entities that can not be categorized as user or company because there is not sufficient contextual information to do so, such as "indians", "third", "individuals" and "americans". Additionally, the reaction or attitude category comprises terms that represent a way of feeling or acting toward a person, thing or situation. For example, the terms "deny" and "admitted" are present in the closest terms to company. A synonymous category emerge during the process as well. This contains equivalent terms to each keyword. For example, the terms "info" and "informations" are close to information and the terms "companys", "corporation", "companies" and "firm" appear among the closest terms to company. Terms with no clear relation to the keywords were classified as undetermined.

We used force-directed graphs (Kobourov, 2013) to represent all the categories that emerged from the analysis of semantic context of each keyword. Figure 3 shows the categories related to the keyword privacy. In this graph, distance represents closeness in the semantic context. For example, terms that were categorized as regulation are closer to privacy than terms that were categorized as security mechanisms. Additionally, the visualization shows examples of terms in each category in Spanish or English. ${ }^{11}$ Force-directed graphs of the categories and terms associated with the other keywords (information, company, and users) are available online. ${ }^{12}$

Overall, we observe that the semantic contexts of four privacy-related keywords include terms corresponding to information privacy concerns. We illustrate such presence in Figure 2. We positioned each IUIPC dimension at the intersection between two of our keywords. Companies carry out collection, handling and or storage activities regarding data \& information. Users exercise (some) agency over the control of their data \& information. The awareness dimension arises from the users' perception of the companies' practices. Our results suggest that the awareness dimension might be further categorized into sub-topics, such as awareness of privacy and security terms, security mechanisms, and privacy and security risks.

Beyond what the IUIPC model proposes, we find that regulations are relevant to Twitter users who talk about Cambridge Analytica. We position this concept close to awareness, as it is considered an environmental factor that relates to information privacy concerns (Lee et al., 2019; Zou et al., 2018; Mohammed and Tejay, 2017) but is not integrated into the IUIPC.

\footnotetext{
11 Terms in Spanish were translated to English by the authors. These terms are shown in cursive

12 https://andreafigue.github.io/word_embeddings/visualization.html
} 


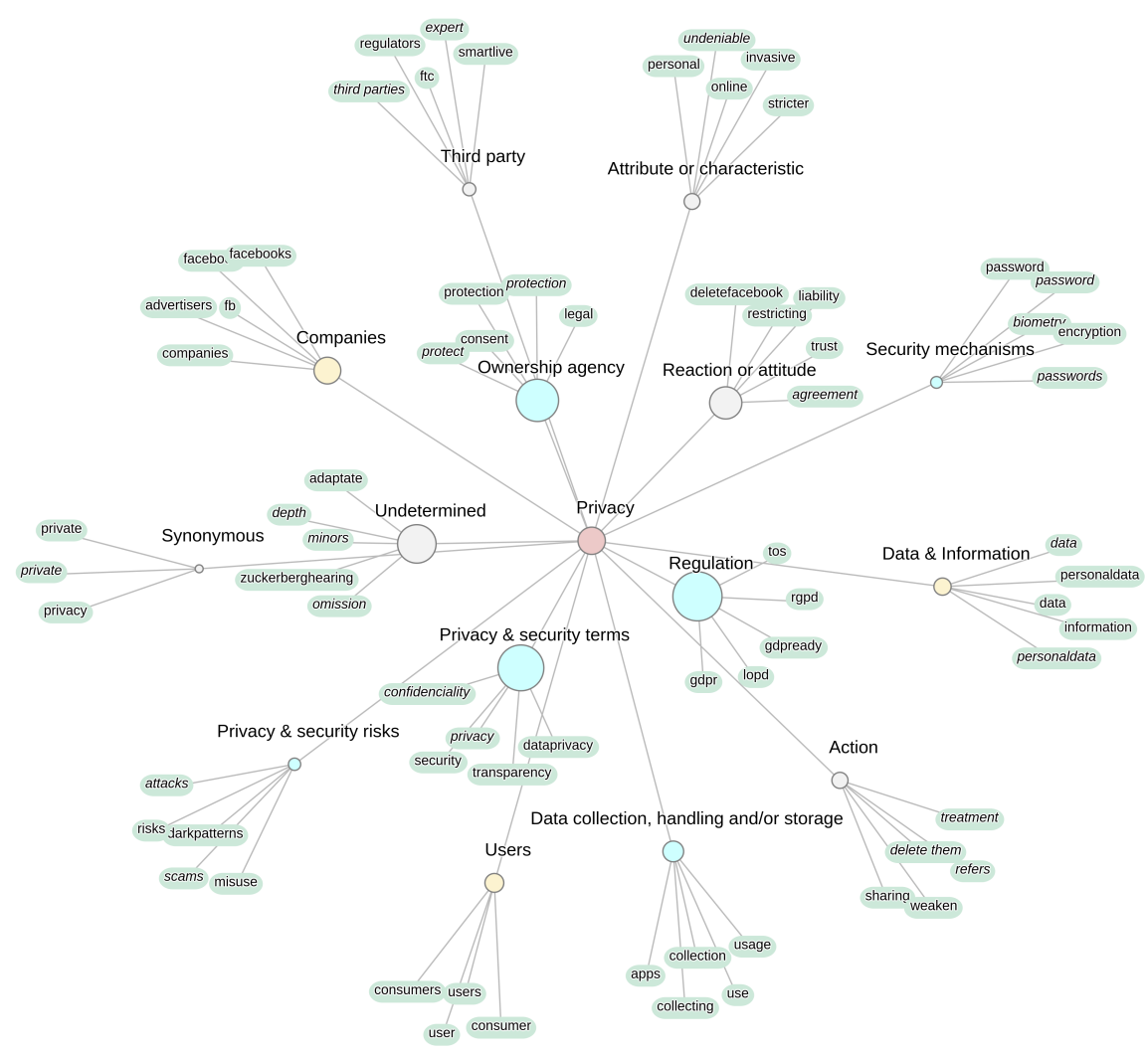

Figure 3. This force-directed graph represents the open coding categories related to the keyword privacy and provides examples of the terms that were coded as each category. Categories with the higher frequency are larger and closer to the keyword. Yellow nodes represent keywords and light-blue nodes denote privacy-related categories.

\subsection{EMPHASIS ON INFORMATION PRIVACY CONCERNS ACROSS LANGUAGES AND WORLD REGIONS}

As we are able to observe the presence of information privacy concerns on the Twitter datasets, we can now turn to test the null hypotheses regarding differences across language and world regions. We compare the emphasis on information privacy concerns (IPC) in the semantic contexts that emerge from the different word embeddings. Figure 4 reports the distribution of terms that relate to the initial keywords (Table $\mathrm{X}$, yellow background) and IPC (Table X, light blue background) in each language and world region under study. Others include all remaining categories.

To test our hypotheses, we performed Chi-square goodness-of-fit tests. Because we ran multiple tests, we applied Šidák correction to counteract the problem of multiple comparisons, thus controlling the family-wise error rate. According to our Šidák 's adjustment, to maintain an overall alpha of 0.05 for the collection of 10 tests, null hypotheses can be rejected when $p<0.0102$.

We find no significant differences on the emphasis on information privacy concerns across languages or regions (see Table XI). Thus, we cannot reject the null hypotheses. We conclude that IPC are present at similar rates in Spanish and English. They cover a considerable proportion of the semantic contexts, with more than $30 \%$ of terms in both languages. Considering the regional datasets, IPC describes between $20 \%$ and $40 \%$ of the terms. While we observe some variation in emphasis on IPC 


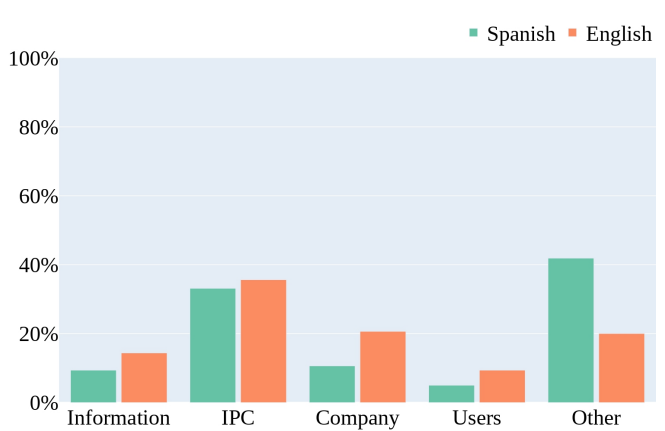

(a) By Language

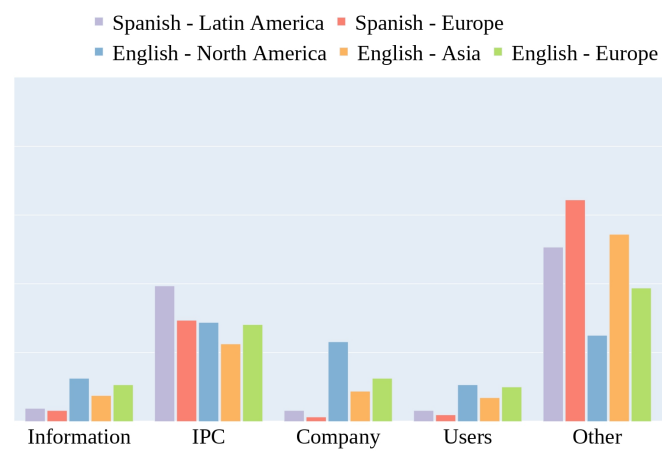

(b) By world-region

Figure 4. Proportion of categories by language (a) and world region (b)

across regions, with the largest proportion in the Latin American dataset and the smallest fraction in the Asian data, the differences across regions are not enough to be statistically significant.

The rest of the terms are better described by our initial categories, such as company, information and users. Compared to the IPC category, all of them cover smaller fractions of the semantic contexts under study. It should also be noted that irrelevant categories (grouped as others) add up to large proportions in all datasets, ranging from $30 \%$ to more than $60 \%$. Together, these results reveal that while a social media dataset about a data breach scandal does bring relevant content about information privacy concerns, this comes with a fair amount of noisy content.

Table XI. Results of Chi-squared tests to compare proportions of terms by language and world regions. The null hypothesis was rejected if $p<.0102$

\begin{tabular}{lrrrr}
\hline \multicolumn{1}{c}{ Null hypothesis } & $\chi^{2}$ & $\mathrm{~N}$ & DF & $p$ value \\
\hline There is no difference in \% of IPC terms between languages & 0.15 & 110 & 1 & .70 \\
There is no difference in \% of IPC terms among world regions & 8.04 & 237 & 4 & .09 \\
\hline There is no difference in \% of collection terms between languages & 11.65 & 31 & 1 & $<.001$ \\
There is no difference in \% of collection terms among world regions & 10.97 & 68 & 4 & .03 \\
There is no difference in \% of control terms between languages & 0.00 & 24 & 1 & 1.00 \\
There is no difference in \% of control terms among world regions & 7.15 & 33 & 4 & .13 \\
There is no difference in \% of awareness terms between languages & 4.12 & 41 & 1 & .04 \\
There is no difference in \% of awareness terms among world regions & 13.58 & 95 & 4 & .009 \\
\hline There is no difference in \% of regulation terms between languages & 0.69 & 13 & 1 & .41 \\
There is no difference in \% of regulation terms among world regions & 11.69 & 26 & 4 & .02 \\
\hline
\end{tabular}

\subsubsection{IUIPC dimensions}

Digging deeper in the terms related to information privacy concerns, we analyze the proportions of terms that match each IUIPC dimension across languages and world regions (see Figure 5 and Table XI).

We observe a broader emphasis on collection in English $\left(\chi^{2}(1,31)=11.65, p=<\right.$ $.001)$ than in Spanish. Cohen's effect size value $(\mathrm{w}=.61)$ suggests that this is a 
high practical significance (Cohen, 1988). Even though this pattern seems to be influenced by a higher proportion on collection in the English content from North America than in any other region (Figure 5), regional differences are not statistically significant after multiple comparisons correction $\left(\chi^{2}(4,68)=10.97, p=.03\right)$.

In turn, while we cannot reject a null hypothesis regarding differences on awareness by language after corrections $\left(\chi^{2}(1,41)=4.12, p=.04\right)$, we find a significant difference across world regions $\left(\chi^{2}(4,95)=13.58, p=.009\right)$. Cohen's effect size value $(\mathrm{w}=.38)$ suggests a moderate to high practical significance. Here, we calculated the standard residuals to determine which world regions make the greater contribution to this chi-square test result. We find that compared with other world regions, data in English from North America have a smaller ratio of awareness terms (chi-square standard residual $=-2.56$ ). The opposite is found in data in Spanish from Latin America (chi-square standard residual $=2.05$ ).

Finally, we find no evidence to reject the null hypothesis regarding control. Control is equally present in both languages and the regions under study.

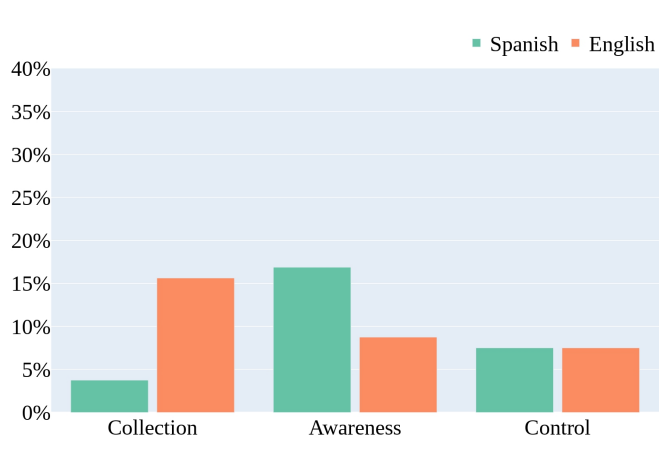

(a) By Language

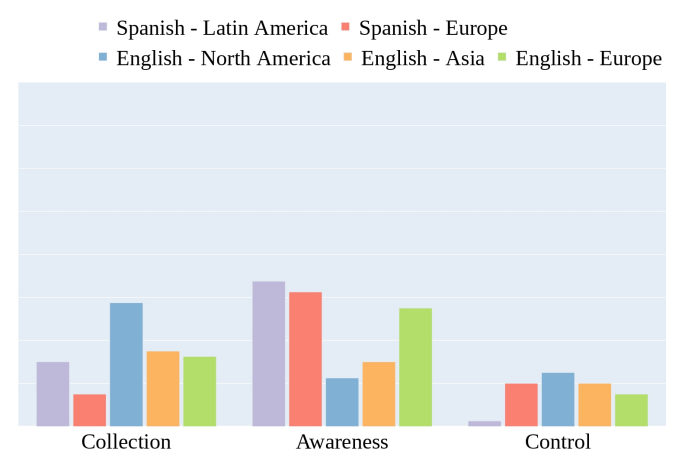

(b) By world-region

Figure 5. Proportion of terms related to the IUIPC dimensions by language (a) and region (b)

\subsubsection{Regulation}

Even though the concept of regulation is not part of the IUIPC dimensions, prior literature (Cockcroft and Rekker, 2016; Lee et al., 2019; Da Veiga, 2018) has suggested that it is related to people's concerns about information privacy. We find terms associated with this category in all our word embeddings (see Figure 6). However, the difference in proportions between Spanish and English data is not statistically significant $\left(\chi^{2}(1,13)=0.69, p=.41\right)$. Likewise, we do not find enough evidence to reject the null hypothesis regarding differences across world regions after multiple comparison correction $\left(\chi^{2}(4,26)=11.69, p=.02\right)$ (see Table XI).

\section{Discussion}

This work explores the potential of social media as a data source to study crosslanguage and cross-regional differences in information privacy concerns. We conduct an analysis of Twitter data related to a particular data breach news to deepen our understanding of how people from different world regions and who speak different languages frame privacy concerns. We chose to focus on the Cambridge Analytica 


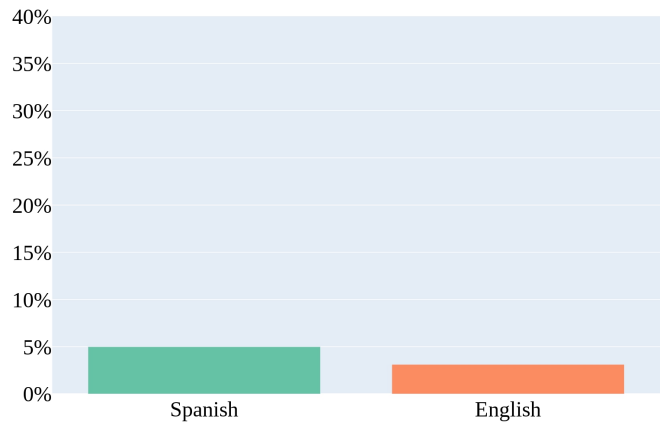

(a) By Language

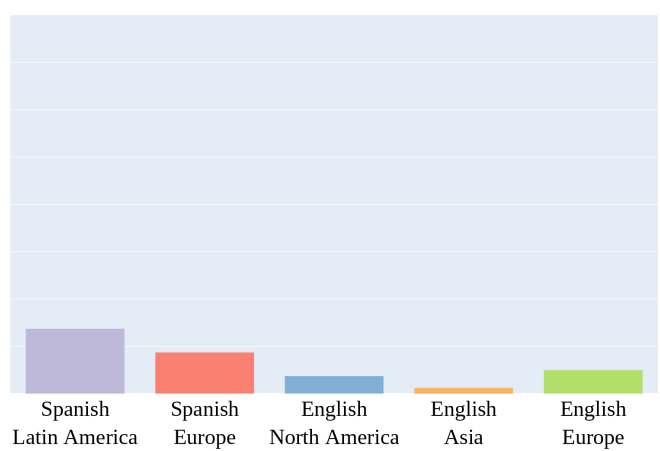

(b) By world-region

Figure 6. Proportion of terms associated to regulations by language (a) and region (b)

scandal because it triggered a wide-ranging exchange on social media about user information and companies' data practices. We build upon the potential of word embeddings to derive a semantic context of each term in a corpus. The contexts are built according to terms that are commonly used in the same phrases. By characterizing a keyword's nearby terms, we seek to reveal the context in which a keyword was discussed (Rho et al., 2018). Based on more than a million non-duplicated, humangenerated tweets, we generate word embeddings for data in Spanish and English and for data from Latin America, North America, Asia and Europe. For each embedding, we conduct a qualitative analysis of the semantic contexts of four privacy-related keywords: information, privacy, company, and users.

Collecting and analyzing the semantic contexts of these privacy-related keywords allows us to observe the presence of terms related to information privacy concerns in the collected tweets. Through iterative manual coding, we characterize the semantic contexts using 15 categories. Several of these categories are easily mapped to the three dimensions of the Internet User Information Privacy Concerns (IUIPC): collection, awareness, and control (See Figure 2). In this way, we find evidence that social media content can reveal information about privacy concerns.

Our approach takes into consideration a vast amount of online content posted freely and spontaneously on Twitter to create the semantic context of each keyword. Thus, it gives a sense of a collective perspective on information privacy concerns by language and world region, which can become a complementary approach to current survey-based methods. Our method aims to discover knowledge from a large-scale social media dataset in a topic for which a ground truth does not exist. Unfortunately, such ground truth is unlikely to exist because large-scale, multi-country, and multi-language surveys are too expensive to conduct ( $\mathrm{Li}$ et al., 2020). As an alternative approach, we used word embeddings to find the semantic contexts of relevant keywords and followed a qualitative approach to validate the results. We carefully analyzed more than a thousand terms of the semantic contexts, conducted open coding to formulate a data-grounded categorization, and then contrasted our categorization with IUIPC (Malhotra et al., 2004), one of the well-accepted theoretical conceptualizations of information privacy concerns. While this is not the common ground truth of other natural language processing tasks such as classification, our process draws from qualitative approaches to validate the results of an automated 
text analysis. We discuss below how our findings extend our current understanding of privacy concerns and open new lines of inquiry.

Beyond matching content to current conceptualizations of information privacy concerns, our results suggest a more granular categorization of one of them. Our results hint that awareness might include more specific sub-topics that users can be aware of, such as privacy and stcurity terms (e.g., cybersecurity, confidentiality),

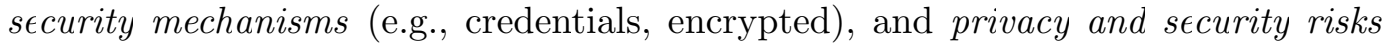
(e.g., scams, grooming). The presence of terms that fit these categories reveals that they are already part of public online conversations around privacy. A distinction among broad privacy and security terms, mechanisms to protect data and potential data risks might be useful to further describe the kinds of knowledge people have. Additionally, awareness about some of these subtopics might be more influential than others. For example, knowing about risks and mechanisms might be a sign of higher privacy concerns while knowing broad privacy and security terms might not. The distinction between sub-topics could also guide users', educators' and practitioners' efforts to enhance information privacy literacy. Future work can explore the relevance of this distinction and its implications for information privacy practices.

Besides, the presence of the regulation category highlights its importance in relation to information privacy concerns. Regulation refers to laws or rules that aim to regulate the use of personal data. They have often been considered a factor influencing information privacy concerns(Ebert et al., 2020; Benamati et al., 2021). The emergence of this category from our open coding confirms its relevance through its frequent appearance in public posts about a data breach scandal. Such relevance might be related to the elaboration of laws and public policies about data usage worldwide. These regulations are not only a topic of data and law experts, but it seems to be part of the public discourse around data privacy online. It is noticeable the common presence of a specific regulation, the GDPR, in our datasets. GDPR is a privacy regulation that has been in effect since late May 2018 in the European Union (Gellman, 2019). Our data collection period covered the early months of its implementation. This regulation prohibits processing and exploiting personal data such as health status, political orientation, sexual preferences, religious beliefs, and ethnic origin. Thus, it aims to decrease the privacy risks that may derive from malicious use of such information, including cases like the Cambridge Analytica scandal (Cabañas et al., 2018). GDPR seeks to convert individuals into empowered citizens involved in the decision-making process related to their personal information (Karampela et al., 2019). As an example, with this regulation in effect, companies are required to inform individuals about their rights (e.g., restriction of processing, erasure of data), the storage period of data, and additional sources that have been used to acquire personal data (Ebert et al., 2020). The explicit presence of GDPR in our data might be evidence of its influence on shaping people's arguments about privacy concerns and its importance not only in Europe but worldwide. Further work can focus on exploring how to integrate better the role of regulations into the current conceptualizations of information privacy concerns, which were proposed long before data privacy regulations were as common as they are now around the globe. Moreover, future work could also explore the interaction between regulations and specific information privacy concerns dimensions.

While we find similar rates of terms related to information privacy concerns across languages and regions, we observe significant differences in emphasis on collection and awareness. These results indicate that different groups view the Cambridge 
Analytica scandal from a particular standpoint. It is important to notice though that while information privacy terms appear through our method, they also come along with a considerable amount of other terms that we consider noisy data. Nevertheless, our findings show the potential of using social media data for cross-language and cross-regional comparisons to identify similarities and nuanced differences on privacy-related perspectives worldwide.

Our analysis reveals that the semantic contexts generated by tweets written in English have significantly more terms related to collection than those written in Spanish. This is a novel finding. When freely expressing online about privacy keywords, English speakers give significantly more emphasis to data collection than Spanish speakers. This difference can lead researchers and practitioners to explore the effectiveness of more tailored data privacy campaigns to specific populations. For example, populations that are more concerned about collection might need more information about the benefits of sharing their information to be able to make a decision about it. A high emphasis on collection in English is also congruent with prior literature observing that college students from the USA are more worried about collection of personal information than control over it (Yang, 2013). Exploring if this trend is shared by people from other English speaking countries can help clarifying which of these patterns are better explained by location or language.

Future work can explore why we observe a significant language difference in emphasis on collection. A feasible explanation might be related to the users' country of residence. Note that our tweets in English come mainly from the USA and UK. Both were the countries most closely connected to the Cambridge Analytica scandal due to the misuse of data for political campaigns in the USA's 2016 presidential election and Brexit (Cadwalladr and Graham-Harrison, 2018). It is possible that this shared experience resulted in a larger emphasis on collection in the English than the Spanish data. An alternative hypothesis is associated with differences in regulations. Information privacy concerns might be a reflection of customer privacy regulations in their respective countries (Markos et al., 2017; Kumar and Reinartz, 2012). In contrast to European countries, that have adopted a data protection directive from a government-imposed perspective, the USA has followed an industry self-regulation (Kumar and Reinartz, 2012). Considering that companies have more freedom to collect and process personal data in North America, it would be reasonable that data collection practices are of deeper concern to individuals from North America than those in Europe. This could also be supported by our data when observing that North America has the highest proportion of terms related to company (see Figure 4), which we also found in our prior work using a different text mining method and a smaller dataset (González et al., 2019b). However, our data analysis does not support the hypothesis of regional differences. It is possible that our data does not have enough power given the multiple comparisons we conducted. Future research is needed to explore alternative hypothesis that can explain the broader emphasis on collection among English speakers, compared to Spanish speakers.

We also observe significant regional differences on awareness. Particularly, data from North America shows the smallest emphasis on awareness while Latin America has the highest. Given that most studies on information privacy concerns are centered on the USA, this finding is particularly important. It warns us against the (sometimes implicit) assumption that North American data about privacy concerns can be generalizable to other regions. At least regarding emphasis on awareness, we find evidence that data from the USA is not similar to other regions. Thus, 
this result provides observational evidence to argue that it is necessary to include more diverse populations to have more a accurate understanding of the phenomena around data privacy. This finding also invites practitioners to address other regions, such as Latin America, using more different approaches in their terms of services and privacy policies. Populations that are more concerned about awareness might be more receptive to companies that use more transparent communications of their use of personal data, for example.

It is worth noting that Latin American shows the largest emphasis on awareness. Our results provide evidence of a disconnection between Latin America and North America regarding this aspect. It is possible that this broad interest on awareness can be a reflection of a connection of Latin America to the European perspective on data privacy. Latin America presents a particular scenario. It lies between two different approaches to personal data regulation: the principles contained by the European GDPR and the fragmented framework of the USA, where data protection is divided by sector (Aguerre, 2019). Privacy regulations are considered an essential concern for many Latin American countries, and after data privacy breaches such as the Cambridge Analytica one, this issue has received increased attention in the public opinion and policy spheres in the region (Aguerre, 2019). Previously, researchers have argued that GDPR could be one of the most influential pieces of data protection legislation ever enacted with influence beyond Europe (Kuner et al., 2017). Indeed, in Brazil, a new GDPR-like law (Lei Geral de Proteção de Dados Pessoais, LGPD, in Portuguese) has become effective since August 2020 (Dias Canedo et al., 2020). Future studies can explore connections among data privacy regulations worldwide, how they relate to public opinion on the issues of privacy, and how they are influenced by national and international data breaches.

As we found regional but not language differences in emphasis on privacy concerns, we conducted a follow-up analysis to assess whether there is a language difference within a single region. Europe was the only region where we had enough data in both languages to conduct such a comparison. We did not find significant differences in emphasis of any IUIPC dimension between data in English and Spanish from Europe $\left(\chi^{2}(3,92)=0.15, p=.98\right)$. There was no evidence of significant differences in emphasis on regulations either. Thus, this additional analysis provides additional evidence to support that information privacy concerns are more related to the region of residence than the spoken language. Nevertheless, further research is required to understand better the role of regulatory regimes, consumer practices, and economic development factors on these differences (Okazaki et al., 2020). As the Spanish-English balance in tweets in our dataset is such that it does not lend itself to intra-region comparison for Asia and North and Latin America, future work could seek to explore if this pattern repeats in those regions as well

As with any study, our research has limitations. We collected data through the free standard streaming Twitter API using specific hashtags and keywords. Thus, we only had access to a limited sample of all the tweets about the scandal. We used Botometer to detect and remove tweets likely to be created by bots. This tool can only analyze Twitter public accounts; therefore, it could not be used on suspended accounts or those with their tweets protected when running our analysis. We decided to remove these accounts' tweets from our datasets because we can not confidently claim that humans generated them. Indeed, previous research suggests that it is likely that social bots were present in this cohort (Heredia et al., 2018). Moreover, we focused our investigation on four keywords in English: information, 
privacy, users, and company and their corresponding translations to Spanish. While using synonyms would have brought similar semantic contexts, adding more concepts can strengthen the results. Future work can explore other keywords such as: intimacy, and consumers. Similarly, we did not use the terms user, and companies as keywords. While word embeddings capture syntactic regularities such as singular/plural forms (Mikolov et al., 2013; Yeşiltaş and Güngör, 2020), we reason that this methodological decision should not have affected considerably our results. Nevertheless, future work could include plural and singular versions of the same term to confirm this hypothesis. The sample size of our manual coding process (40 words per keyword in each embedding) could have impacted the results. We chose the number of retrieved terms after manually inspecting the list of nearest words by each keyword in all our embeddings. We picked a threshold that allowed us to obtain a high number of meaningful words in most embeddings. Higher thresholds make it more likely to include terms with no apparent relation to the keywords (e.g., v.i.p; ground; approximately). In word embeddings with reduced vocabularies like ours, the number of relevant terms available for a specific keyword is limited. This characteristic explains why the number of irrelevant terms (Other in Figure 3) is high in datasets with small vocabularies, such as the Spanish and English-Asia datasets. Future work could evaluate how sensitive our approach is to changes in vocabulary size and threshold for the nearest terms. This decision may introduce a bias in the results, and it is one of the limitations of our approach of social media textual data.

\section{Conclusion}

We conducted a cross-language and cross-regional study on social media content about a major data privacy leakage: the Cambridge Analytica scandal. We categorized our Twitter data into two different languages and four geographical regions. Our results shed light on language and regional differences on information privacy concerns by 1) creating word embeddings by language and world regions to leverage social media data about a data breach scandal, 2) conducting open coding and content analysis of the semantic contexts (generated by the embeddings) of privacy-related keywords, 3) mapping the results to a well-known information privacy framework, and (4) conducting a comparative analysis across two languages and four world regions.

We found that data in English shows a broader emphasis on data collection, while data from North America shows the smallest emphasis on awareness. In turn, data from Latin America has the broadest emphasis on awareness. We discuss how our findings extend current conceptualizations of information privacy concerns, and how they might relate to regulations about personal data usage in the regions we analyzed.

Future work can dig deeper on the differences we observed and explore further the potential causes we discussed. Future studies might build upon our work to examine privacy concerns considering more languages, more geographical locations or different information privacy frameworks. Using our methodology to compare datasets across longer periods of time could be useful to determine if the semantic contexts of the privacy keywords changes over time. 


\section{Acknowledgments}

The authors want to thank Francisco Tobar, MSc. Computer Science student at Universidad Técnica Federico Santa María, for helping us to strengthen our findings through statistical analysis. Moreover, we acknowledge anonymous reviewers for insightful comments that helped us revise and refine the paper.

\section{Funding and conflicts of interests}

This collaboration was possible thanks to the support of the Fulbright Program, under a 2017-18 Fulbright Fellowship award. This work was also partially funded by CONICYT Chile, under grant Conicyt-Fondecyt Iniciación 11161026. The first author acknowledges the support of the PIIC program from Universidad Técnica Federico Santa María and CONICYT-PFCHA/MagísterNacional/2019-22190332. The authors declare that there is no conflict of interest regarding the publication of this paper.

\section{References}

Adu, Ernest K; Nelly Todorova; and Annette Mills (2019). Do Individuals in Developing Countries Care about Personal Health Information Privacy? An Empirical Investigation. In CONF-IRM 2019. Proceedings of the 2019 International Conference on Information Resources Management, Auckland, New Zealand, 27-29 May, 2019. Auckland, New Zealand: School of Business, University of Auckland, p. 16.

Aghababaei, Somayyeh; and Masoud Makrehchi (2017). Activity-based Twitter sampling for content-based and user-centric prediction models. Human-centric Computing and Information Sciences, vol. 7 , no. 1, p. 3.

Agüero-Torales, Marvin M; David Vilares; and Antonio G López-Herrera (2021). Discovering topics in Twitter about the COVID-19 outbreak in Spain. Procesamiento del Lenguaje Natural, vol. 66, pp. $177-190$.

Aguerre, Carolina (2019). Digital trade in Latin America: mapping issues and approaches. Digital Policy, Regulation and Governance, vol. 21, no. 1, pp. 2-18.

Badawy, Adam; Emilio Ferrara; and Kristina Lerman (2018). Analyzing the digital traces of political manipulation: the 2016 Russian interference Twitter campaign. In ASONAM 2018. Proceedings of the IEEE/ACM International Conference on Advances in Social Networks Analysis and Mining, Barcelona, Spain, 28-31 August, 2018. IEEE, pp. 258-265.

Bélanger, France; and Robert E Crossler (2011). Privacy in the digital age: a review of information privacy research in information systems. Management Information Systems Quarterly. MIS quarterly, vol. 35, no. 4, pp. 1017-1042.

Bellman, Steven; Eric J Johnson; Stephen J Kobrin; and Gerald L Lohse (2004). International differences in information privacy concerns: A global survey of consumers. The Information Society, vol. 20, no. 5, pp. 313-324.

Benamati, John H.; Zafer D. Ozdemir; and H. Jeff Smith (2021). Information Privacy, Cultural Values, and Regulatory Preferences. Journal of Global Information Management (JGIM), vol. 29, no. 3, pp. 131-164.

Bergsma, Shane; Mark Dredze; Benjamin Van Durme; Theresa Wilson; and David Yarowsky (2013). Broadly improving user classification via communication-based name and location clustering on twitter. In NAACL HLT 2013. Proceedings of the 2013 Conference of the North American Chapter of the Association for Computational Linguistics: Human Language Technologies, Atlanta, Georgia, USA, 9-14 June, 2013. Association for Computational Linguistics, pp. 1010-1019.

Bolukbasi, Tolga; Kai-Wei Chang; James Y Zou; Venkatesh Saligrama; and Adam T Kalai (2016). Man is to computer programmer as woman is to homemaker? debiasing word embeddings. In NIPS 2016. Advances in Neural Information Processing Systems, Barcelona, Spain, 5-10 December, 2016. NY, United States: Curran Associates Inc., pp. 4349-4357. 
Buchanan, Tom; Carina Paine; Adam N Joinson; and Ulf-Dietrich Reips (2007). Development of measures of online privacy concern and protection for use on the Internet. Journal of the American Society for Information Science and Technology, vol. 58, no. 2, pp. 157-165.

C. Sipior, Janice; Burke T. Ward; and Regina Connolly (2013). Empirically assessing the continued applicability of the IUIPC construct. Journal of Enterprise Information Management, vol. 26, no. 6, pp. 661-678.

Cabañas, José González; Ángel Cuevas; and Rubén Cuevas (2018). Unveiling and Quantifying Facebook Exploitation of Sensitive Personal Data for Advertising Purposes. In SEC'18. Proceedings of the 27th USENIX Conference on Security Symposium. USENIX Association, p. 479-495.

Cadwalladr, Carole; and Emma Graham-Harrison (2018). Revealed: 50 million Facebook profiles harvested for Cambridge Analytica in major data breach. http://www.theguardian . com/news/2018/mar/17/cambridge-analytica-facebook-influence-us-election. Accessed 16 July 2020.

Cannataci, Joseph A. (2009). Privacy, Technology Law and Religions across Cultures. Journal of Information, Law and Technology, vol. 2009, no. 1.

Cao, Jinwei; and Andrea Everard (2008). User attitude towards instant messaging: The effect of espoused national cultural values on awareness and privacy. Journal of Global Information Technology Management, vol. 11, no. 2, pp. 30-57.

Chai, Sangmi (2020). Does Cultural Difference Matter on Social Media? An Examination of the Ethical Culture and Information Privacy Concerns. Sustainability, vol. 12, no. 19.

Cho, Hichang; Milagros Rivera-Sánchez; and Sun Sun Lim (2009). A multinational study on online privacy: global concerns and local responses. New media 83 society, vol. 11, no. 3, pp. 395-416.

Cockcroft, Sophie; and Saphira Rekker (2016). The relationship between culture and information privacy policy. Electronic Markets, vol. 26, no. 1, pp. 55-72.

Cohen, Jacob (1988). Statistical Power Analysis for the Behavioral Sciences. Hillsdale, N.J.: L. Erlbaum Associates.

Consolvo, Sunny; Ian E Smith; Tara Matthews; Anthony LaMarca; Jason Tabert; and Pauline Powledge (2005). Location disclosure to social relations: why, when, \& what people want to share. In CHI'05. Proceedings of the SIGCHI conference on Human factors in computing systems, Portland, Oregon, USA, 2-7 April, 2005. New York, NY, USA: Association for Computing Machinery, pp. 81-90.

Correia, John; and Deborah Compeau (2017). Information Privacy Awareness (IPA): A Review of the Use, Definition and Measurement of IPA. In HICSS-50. Proceedings of the 50th Hawaii International Conference on System Sciences, Hilton Waikoloa Village, Big Island, HI, USA, 4-7 January, 2017. ScholarSpace / AIS Electronic Library (AISeL).

Da Veiga, Adéle (2018). An information privacy culture instrument to measure consumer privacy expectations and confidence. Information $\mathcal{E}$ Computer Security, vol. 26, no. 3, pp. 338-364.

Davis, Clayton Allen; Onur Varol; Emilio Ferrara; Alessandro Flammini; and Filippo Menczer (2016). Botornot: A system to evaluate social bots. In $W W W$ '16 Companion. Proceedings of the 25th International Conference Companion on World Wide Web, Montréal, Québec, Canada, 11-15 April, 2016. International World Wide Web Conferences Steering Committee, pp. 273-274.

Dias Canedo, Edna; Angelica Toffano Seidel Calazans; Eloisa Toffano Seidel Masson; Pedro Henrique Teixeira Costa; and Fernanda Lima (2020). Perceptions of ICT Practitioners Regarding Software Privacy. Entropy, vol. 22, no. 4, p. 429.

Dienlin, Tobias; and Sabine Trepte (2015). Is the privacy paradox a relic of the past? An in-depth analysis of privacy attitudes and privacy behaviors. European journal of social psychology, vol. 45 , no. 3, pp. $285-297$.

Dinev, Tamara; and Paul Hart (2006). An extended privacy calculus model for e-commerce transactions. Information systems research, vol. 17, no. 1, pp. 61-80.

Ebert, Nico; Kurt Alexander Ackermann; and Peter Heinrich (2020). Does Context in Privacy Communication Really Matter? - A Survey on Consumer Concerns and Preferences. In CHI'20. Proceedings of the 2020 CHI Conference on Human Factors in Computing Systems, Honolulu, HI, USA, 25-30 April, 2020. New York, NY, USA: Association for Computing Machinery, pp. $1-11$.

Egelman, Serge; and Eyal Peer (2015a). Predicting privacy and security attitudes. ACM Special Interest Group on Computers and Society. SIGCAS, vol. 45, no. 1, pp. 22-28.

Egelman, Serge; and Eyal Peer (2015b). Scaling the security wall: Developing a security behavior intentions scale (sebis). In CHI'15. Proceedings of the 33rd Annual ACM Conference on Human 
Factors in Computing Systems, Seoul, Republic of Korea, 18-23 April, 2015. New York, NY, USA: Association for Computing Machinery, pp. 2873-2882.

Gellman, Robert (2019). Fair Information Practices: A Basic History-Version 2.19. Social Science Research Network. SSRN Electronic Journal. https://papers.ssrn.com/sol3/papers.cfm? abstract_id=2415020.

Gerber, Nina; Paul Gerber; and Melanie Volkamer (2018). Explaining the privacy paradox: A systematic review of literature investigating privacy attitude and behavior. Computers $\&$ Security, vol. 77 , pp. 226-261.

González, Felipe; Andrea Figueroa; Claudia López; and Cecilia Aragon (2019a). Information Privacy Opinions on Twitter: A Cross-Language Study. In CSCW '19. Conference Companion Publication of the 2019 on Computer Supported Cooperative Work and Social Computing, Austin, TX, USA, 9-13 November, 2019. New York, NY, USA: Association for Computing Machinery, CSCW'19, pp. 190-194.

González, Felipe; Yihan Yu; Andrea Figueroa; Claudia López; and Cecilia Aragon (2019b). Global Reactions to the Cambridge Analytica Scandal: A Cross-Language Social Media Study. In $W W W$ '19. Companion Proceedings of The 2019 World Wide Web Conference, San Francisco, USA, 13-17 May, 2019. New York, NY, USA: Association for Computing Machinery, pp. 799-806.

Hajjem, Malek; and Chiraz Latiri (2017). Combining IR and LDA Topic Modeling for Filtering Microblogs. Procedia Computer Science, vol. 112, pp. 761-770.

Harborth, David; and Sebastian Pape (2017). Privacy concerns and behavior of Pokémon go players in Germany. In 12th IFIP Summer School on Privacy and Identity Management, Ispra, Italy, 3-8 September, 201\%. Springer, pp. 314-329.

Harborth, David; and Sebastian Pape (2018). German Translation of the Concerns for Information Privacy (CFIP) Construct. Social Science Research Network. SSRN Electronic Journal. https: //ssrn. com/abstract=3112207.

Harzing, Anne-Wil (2005). Does the use of English-language questionnaires in cross-national research obscure national differences? International Journal of Cross Cultural Management, vol. 5, no. 2, pp. 213-224.

Harzing, Anne-Wil (2006). Response styles in cross-national survey research: A 26-country study. International Journal of Cross Cultural Management, vol. 6, no. 2, pp. 243-266.

Harzing, Anne-Wil; and Martha Maznevski (2002). The interaction between language and culture: A test of the cultural accommodation hypothesis in seven countries. Language and Intercultural Communication, vol. 2, no. 2, pp. 120-139.

Haynes, Winston (2013). Bonferroni Correction, New York, NY: Springer New York, pp. 154-154.

Heravi, Alireza; Sameera Mubarak; and Kim-Kwang Raymond Choo (2018). Information privacy in online social networks: Uses and gratification perspective. Computers in Human Behavior, vol. 84, pp. 441-459.

Heredia, Brian; Joseph D. Prusa; and Taghi M. Khoshgoftaar (2018). The Impact of Malicious Accounts on Political Tweet Sentiment. In CIC'18. Proceedings of the 4th International Conference on Collaboration and Internet Computing, Philadelphia, PA, USA, 18-20 October, 2018. Philadelphia, PA: IEEE, pp. 197-202.

Hofstede, Geert (1983). National cultures in four dimensions: A research-based theory of cultural differences among nations. International Studies of Management $\&$ Organization, vol. 13, no. 1-2, pp. 46-74.

Hofstede, Geert (2011). Dimensionalizing cultures: The Hofstede model in context. Online readings in psychology and culture, vol. 2, article 8, 26 pages.

Hong, Weiyin; and James Y. L. Thong (2013). Internet Privacy Concerns: An Integrated Conceptualization and Four Empirical Studies. Management Information Systems Quarterly. MIS Quarterly, vol. 37, no. 1, pp. 275-298.

Huang, Hsiao-Ying; and Masooda Bashir (2016). Privacy by region: Evaluation online users' privacy perceptions by geographical region. In FTC 2016. Future Technologies Conference, San Francisco, California, USA, 6-7 December, 2016. IEEE, pp. 968-977.

Hussein, Basel Al-Sheikh (2012). The sapir-whorf hypothesis today. Theory and Practice in Language Studies, vol. 2, no. 3, pp. 642-646.

Ilyas, Sardar Haider Waseem; Zainab Tariq Soomro; Ahmed Anwar; Hamza Shahzad; and Ussama Yaqub (2020). Analyzing Brexit's Impact Using Sentiment Analysis and Topic Modeling on Twitter Discussion. In DG.O'20. Proceedings of the 21st Annual International Conference on Digital Government Research, Seoul, Republic of Korea, 17-19 June, 2020. New York, NY, USA: Association for Computing Machinery, pp. 1-6. 
Isaak, Jim; and Mina J Hanna (2018). User Data Privacy: Facebook, Cambridge Analytica, and Privacy Protection. Computer, vol. 51, no. 8, pp. 56-59.

Jackoway, Alan; Hanan Samet; and Jagan Sankaranarayanan (2011). Identification of live news events using Twitter. In LBSN '11. Proceedings of the 3rd ACM SIGSPATIAL International Workshop on Location-Based Social Networks, Chicago, Illinois, USA, 1 November, 2011. New York, NY, USA: Association for Computing Machinery, pp. 25-32.

Jai, Tun-Min Catherine; and Nancy J King (2016). Privacy versus reward: Do loyalty programs increase consumers' willingness to share personal information with third-party advertisers and data brokers? Journal of Retailing and Consumer Services, vol. 28, pp. 296-303.

Jha, Akshita; and Radhika Mamidi (2017). When does a compliment become sexist? analysis and classification of ambivalent sexism using twitter data. In Proceedings of the Second Workshop on NLP and Computational Social Science, Vancouver, Canada, August, 2017. Association for Computational Linguistics, pp. 7-16.

Jiang, Ke; Grace A. Benefield; Junfei Yang; and George Barnett (2017). Mapping Articles on China in Wikipedia An Inter-Language Semantic Network Analysis. In HICSS-50. Proceedings of the 50th Hawaii International Conference on System Sciences, Hilton Waikoloa Village, Big Island, HI, USA, 4-7 January, 2017. ScholarSpace / AIS Electronic Library (AISeL), pp. 2233-2242.

Jozani, Mohsen; Emmanuel Ayaburi; Myung Ko; and Kim-Kwang Raymond Choo (2020). Privacy concerns and benefits of engagement with social media-enabled apps: A privacy calculus perspective. Computers in Human Behavior, vol. 107, article 106260, 15 pages.

Karampela, Maria; Sofia Ouhbi; and Minna Isomursu (2019). Exploring users' willingness to share their health and personal data under the prism of the new GDPR: implications in healthcare. In EMBC 2019. Proceedings of the 41st Annual International Conference of the IEEE Engineering in Medicine and Biology Society, Berlin, Germany, 23-27 July, 2019. IEEE, pp. 6509-6512.

Kay, Paul; and Willett Kempton (1984). What is the Sapir-Whorf hypothesis? American anthropologist, vol. 86, no. 1, pp. 65-79.

Kobourov, Stephen G. (2013). Force-Directed Drawing Algorithms. In Roberto Tamassia (ed.), Handbook of Graph Drawing and Visualization, Chapman and Hall/CRC, pp. 383-408.

Kokolakis, Spyros (2017). Privacy attitudes and privacy behaviour: A review of current research on the privacy paradox phenomenon. Computers \& security, vol. 64, pp. 122-134.

Krasnova, Hanna; and Natasha F Veltri (2010). Privacy calculus on social networking sites: Explorative evidence from Germany and USA. In HICSS'10. Proceedings of the $201043 \mathrm{rd}$ Hawaii International Conference on System Sciences, Koloa, Kauai, HI, USA, 5-8 January, 2010. Washington, DC, USA.: IEEE, pp. 1-10.

Kumar, V.; and Werner Reinartz (2012). Customer Privacy Concerns and Privacy Protective Responses, Berlin, Heidelberg: Springer, pp. 279-300.

Kumaraguru, Ponnurangam; and Lorrie Faith Cranor (2005). Privacy indexes: a survey of Westin's studies. Tech. Rep. CMU-ISRI-5-138, Institute for Software Research International, School of Computer Science, Carnegie Mellon University, Pittsburgh, PA. https://www.cs.cmu.edu/ ponguru/CMU-ISRI-05-138.pdf. Accessed 16 July 2020.

Kuner, Christopher; Dan Jerker; B Svantesson; Fred H Cate; Orla Lynskey; Christopher Millard; and Nora Ni Loideain (2017). The GDPR as a chance to break down borders. International Data Privacy Law, vol. 7, no. 4, pp. 231-232.

Lagos, Vasileios (2017). Comparative analysis of the hashtags \#RefugeesWelcome and \#StopRefugees: Tweets mining and examination with the Knime Analytics Platform. Master's thesis, University of Peloponnese.

Lapaire, Jean-Rémi (2018). Why content matters. Zuckerberg, Vox Media and the Cambridge Analytica data leak. ANTARES: Letras e Humanidades, vol. 10, no. 20, pp. 88-110.

Lee, Hwansoo; Dongwon Lim; Hyerin Kim; Hangjung Zo; and Andrew P Ciganek (2015). Compensation paradox: the influence of monetary rewards on user behaviour. Behaviour $\&$ Information Technology, vol. 34, no. 1, pp. 45-56.

Lee, Hwansoo; Siew Fan Wong; Jungjoo Oh; and Younghoon Chang (2019). Information privacy concerns and demographic characteristics: Data from a Korean media panel survey. Government Information Quarterly, vol. 36, no. 2, pp. 294-303.

Leetaru, Kalev (2019). Is Twitter's Spritzer Stream Really A Nearly Perfect 1\% Sample Of Its Firehose?, Forbes. https://www.forbes.com/sites/kalevleetaru/2019/02/27/is-twittersspritzer-stream-really-a-nearly-perfect-1-sample-of-its-firehose/. Accessed 16 July 2020 . 
Leidner, Dorothy E; and Timothy Kayworth (2006). A review of culture in information systems research: Toward a theory of information technology culture conflict. Management Information Systems Quarterly. MIS quarterly, vol. 30, no. 2, pp. 357-399.

Li, Yao; Alfred Kobsa; Bart P Knijnenburg; and MH Carolyn Nguyen (2017). Cross-cultural privacy prediction. Proceedings on Privacy Enhancing Technologies, vol. 2017, no. 2, pp. 113-132.

Li, Yao; Eugenia Ha Rim Rho; and Alfred Kobsa (2020). Cultural differences in the effects of contextual factors and privacy concerns on users' privacy decision on social networking sites. Behaviour \& Information Technology, pp. 1-23.

Lin, Yu-Wei (2018). \#DeleteFacebook is still feeding the beast-but there are ways to overcome surveillance capitalism, The Conversation Trust. https://theconversation. com/deletefacebook-is-still-feeding-the-beast-but-there-are-ways-to-overcomesurveillance-capitalism-93874. Accessed 16 July 2020.

Liu, Dapeng; and Lemuria Carter (2018). Impact of citizens' privacy concerns on e-government adoption. In DG-O'18. Proceedings of the 19th Annual International Conference on Digital Government Research: Governance in the Data Age, Delft, The Netherlands, 30 May - 1 June, 2018. pp. 1-6.

Malhotra, Naresh K; Sung S Kim; and James Agarwal (2004). Internet users' information privacy concerns (IUIPC): The construct, the scale, and a causal model. Information systems research, vol. 15, no. 4, pp. 336-355.

Markos, Ereni; George R Milne; and James W Peltier (2017). Information sensitivity and willingness to provide continua: a comparative privacy study of the United States and Brazil. Journal of Public Policy \& Marketing, vol. 36, no. 1, pp. 79-96.

Mikolov, Tomas; Ilya Sutskever; Kai Chen; Greg S Corrado; and Jeff Dean (2013). Distributed representations of words and phrases and their compositionality. In NIPS 2013. Advances in neural information processing systems, Lake Tahoe, Nevada, USA, 5-10 December, 2013. pp. 3111-3119.

Mirchandani, Maya (2018). To delete, or not to \#deleteFacebook, that is the question, The Wire. https://thewire.in/media/to-delete-or-not-to-deletefacebook-that-isthe-question. Accessed 16 July 2020.

Mohammed, Zareef A.; and Gurvirender P. Tejay (2017). Examining Privacy Concerns and Ecommerce Adoption in Developing Countries. Computers $\& 5$ Security, vol. 67, no. C, pp. $254-265$.

Morstatter, Fred; Jürgen Pfeffer; and Huan Liu (2014). When is it biased?: assessing the representativeness of twitter's streaming API. In WWW'14. Proceedings of the 23rd international conference on world wide web, Seoul, Korea, 7-11 April, 2014. New York, NY, USA: Association for Computing Machinery, pp. 555-556.

Morstatter, Fred; Jürgen Pfeffer; Huan Liu; and Kathleen M Carley (2013). Is the sample good enough? comparing data from twitter's streaming api with twitter's firehose. In ICWSM2013. Proceedings of the Seventh International AAAI Conference on Weblogs and Social Media, Cambridge, Massachusetts, USA, 8-11 July, 2013. Menlo Park, California: AAAI Press, pp. 400-408.

Morton, Anthony; and M Angela Sasse (2014). Desperately seeking assurances: Segmenting users by their information-seeking preferences. In PST 2014. Proceedings of the 12th International Conference on Privacy, Security and Trust, Toronto, Canada, 23-24 July, 2014. IEEE, pp. $102-111$.

Motiwalla, Luvai F; Xiaobai (Bob) Li; and Xiaoping Liu (2014). Privacy paradox: Does stated privacy concerns translate into the valuation of personal information? In PACIS 2014. Proceedings of the 18th Pacific Asia Conference on Information Systems, Chengdu, China, 24-28 June, 2014. p. 281. Article 281.

Newell, Patricia Brierley (1995). Perspectives on privacy. Journal of environmental psychology, vol. 15, no. 2, pp. 87-104.

Nov, Oded; and Sunil Wattal (2009). Social computing privacy concerns: antecedents and effects. In CHI'09. Proceedings of the SIGCHI conference on human factors in computing systems, Boston, MA, USA, 4-9 April, 2009. New York, NY, USA: Association for Computing Machinery, pp. 333-336.

O'Connor, Brendan; Ramnath Balasubramanyan; Bryan R Routledge; and Noah A Smith (2010). From tweets to polls: Linking text sentiment to public opinion time series. In ICWSM-2010. Proceedings of the fourth international AAAI conference on weblogs and social media, Washington DC, USA, 23-26 May, 2010. Menlo Park, California: AAAI Press, pp. 122-129. 
Oghazi, Pejvak; Rakel Schultheiss; Koteshwar Chirumalla; Nicolas Philipp Kalmer; and Fakhreddin F. Rad (2020). User self-disclosure on social network sites: A cross-cultural study on Facebook's privacy concepts. Journal of Business Research, vol. 112, pp. 531-540.

Okazaki, Shintaro; Martin Eisend; Kirk Plangger; Ko de Ruyter; and Dhruv Grewal (2020). Understanding the Strategic Consequences of Customer Privacy Concerns: A Meta-Analytic Review. Journal of Retailing, vol. 96, no. 4, pp. 458-473.

Palos-Sanchez, Pedro; José Hernandez-Mogollon; and Ana Campon-Cerro (2017). The behavioral response to location based services: An examination of the influence of social and environmental benefits, and privacy. Sustainability, vol. 9, no. 11, article 1988.

Raber, Frederic; and Antonio Krüger (2018). Privacy Perceiver: Using Social Network Posts to Derive Users' Privacy Measures. In UMAP'18. Adjunct Publication of the 26th Conference on User Modeling, Adaptation and Personalization, Singapore, Singapore, 8-11 July, 2018. New York, NY, USA: Association for Computing Machinery, pp. 227-232.

Rho, Eugenia Ha Rim; Gloria Mark; and Melissa Mazmanian (2018). Fostering Civil Discourse Online: Linguistic Behavior in Comments of \#MeToo Articles across Political Perspectives. In $C S C W$ 2018. Proceedings of the ACM on Human-Computer Interaction. Association for Computing Machinery, vol. 2, article 147, 28 pages.

Rowan, Mark; and Josh Dehlinger (2014). Observed gender differences in privacy concerns and behaviors of mobile device end users. Procedia Computer Science, vol. 37, pp. 340-347.

Schneble, Christophe Olivier; Bernice Simone Elger; and David Shaw (2018). The Cambridge Analytica affair and Internet-mediated research. European Molecular Biology Organization. EMBO reports, vol. 19, no. 8, article e46579.

Shen, Chien-Wen; and Chin-Jin Kuo (2014). Analysis of social influence and information dissemination in social media: The case of Twitter. In CISIM. Proceedings of the 13th IFIP International Conference on Computer Information Systems and Industrial Management, Ho Chi Minh City, Vietnam, November 2014. Berlin, Heidelberg: Springer Berlin Heidelberg, pp. 526-534.

Singh, Loitongbam Gyanendro; Akash Anil; and Sanasam Ranbir Singh (2020). SHE: Sentiment Hashtag Embedding Through Multitask Learning. IEEE Transactions on Computational Social Systems, vol. 7, no. 2, pp. 417-424.

Smith, H Jeff; Tamara Dinev; and Heng Xu (2011). Information privacy research: an interdisciplinary review. Management Information Systems Quarterly. MIS quarterly, vol. 35, no. 4, pp. 989-1016.

Smith, H Jeff; Sandra J Milberg; and Sandra J Burke (1996). Information privacy: measuring individuals' concerns about organizational practices. Management Information Systems Quarterly. MIS quarterly, pp. 167-196.

Solon, Olivia; and Oliver Laughland (2018). Cambridge Analytica closing after Facebook data harvesting scandal, The Guardian. https://www.theguardian.com/uk-news/2018/may/02/ cambridge-analytica-closing-down-after-facebook-row-reports-say. Accessed 16 July 2020.

Stewart, Kathy A; and Albert H Segars (2002). An empirical examination of the concern for information privacy instrument. Information systems research, vol. 13, no. 1, pp. 36-49.

Tavoschi, Lara; Filippo Quattrone; Eleonora D'Andrea; Pietro Ducange; Marco Vabanesi; Francesco Marcelloni; and Pier Luigi Lopalco (2020). Twitter as a sentinel tool to monitor public opinion on vaccination: an opinion mining analysis from September 2016 to August 2017 in Italy. Human Vaccines ES Immunotherapeutics, vol. 16, no. 5, pp. 1062-1069. PMID: 32118519.

Terlutter, Ralf; Sandra Diehl; and Barbara Mueller (2006). The GLOBE study -applicability of a new typology of cultural dimensions for cross-cultural marketing and advertising research. International advertising and communication, pp. 419-438.

Torabi, Sadegh; and Konstantin Beznosov (2016). Sharing Health Information on Facebook: Practices, Preferences, and Risk Perceptions of North American Users. In SOUPS 2016. Twelfth Symposium on Usable Privacy and Security. USENIX Association, pp. 301-320.

Tumasjan, Andranik; Timm O Sprenger; Philipp G Sandner; and Isabell M Welpe (2010). Predicting elections with twitter: What 140 characters reveal about political sentiment. In ICWSM2010. Proceedings of the Fourth international AAAI conference on weblogs and social media, Washington DC, USA, 23-26 May, 2010. Menlo Park, California: AAAI Press.

United Nations Conference on Trade and Development (2020). Data Protection and Privacy Legislation Worldwide. https://unctad.org/en/Pages/DTL/STI_and_ICTs/ICT4D-Legislation/ eCom-Data-Protection-Laws . aspx. Accessed 12 February 2020. 
Ur, Blase; and Yang Wang (2013). A cross-cultural framework for protecting user privacy in online social media. In $W W W$ '13. Proceedings of the 22nd International Conference on World Wide Web, Rio de Janeiro, Brazil, 13-17 May, 2013. New York, NY, USA: Association for Computing Machinery, pp. 755-762.

Van Slyke, Craig; JT Shim; Richard Johnson; and James J Jiang (2006). Concern for information privacy and online consumer purchasing. Journal of the Association for Information Systems, vol. 7 , no. 6 , pp. $415-444$.

Varol, Onur; Emilio Ferrara; Clayton A Davis; Filippo Menczer; and Alessandro Flammini (2017). Online human-bot interactions: Detection, estimation, and characterization. In ICWSM-201\%. Proceedings of the eleventh international AAAI conference on web and social media, Montréal, Québec, Canada, 15-18 May, 2017. Palo Alto, California: AAAI Press, pp. 280-289.

Venturini, Tommaso; and Richard Rogers (2019). "API-Based Research" or How can Digital Sociology and Journalism Studies Learn from the Facebook and Cambridge Analytica Data Breach. Digital Journalism, pp. 1-9.

Viera, Anthony J; Joanne M Garrett; et al. (2005). Understanding interobserver agreement: the kappa statistic. Family Medicine, vol. 37, no. 5, pp. 360-363.

Vitak, Jessica; Stacy Blasiola; Sameer Patil; and Eden Litt (2015). Balancing Audience and Privacy Tensions on Social Network Sites: Strategies of Highly Engaged Users. International Journal of Communication, vol. 9, pp. 1485-1504.

Vitkauskaite, Elena (2010). Overview of research on cross-cultural impact on social networking sites. Economics and Management, vol. 15, pp. 844-848.

Wang, Bin; Angela Wang; Fenxiao Chen; Yuncheng Wang; and C.-C. Jay Kuo (2019). Evaluating word embedding models: methods and experimental results. APSIPA Transactions on Signal and Information Processing, vol. 8, article e19, 14 pages.

Wang, Haizhou; and Mingzhou Song (2011). Ckmeans. 1d. dp: optimal k-means clustering in one dimension by dynamic programming. The $R$ journal, vol. 3, no. 2, pp. 29-33.

Williams, Michael; and Tami Moser (2019). The art of coding and thematic exploration in qualitative research. International Management Review, vol. 15, no. 1, pp. 45-55.

Wisniewski, Pamela; A.K.M. Najmul Islam; Bart P. Knijnenburg; and Sameer Patil (2015). Give Social Network Users the Privacy They Want. In CSCW'15. Proceedings of the 18th ACM Conference on Computer Supported Cooperative Work 85 Social Computing, Vancouver, BC, Canada, 14-18 March, 2015. New York, NY, USA: Association for Computing Machinery, pp. 1427-1441.

Wisniewski, Pamela J.; Bart P. Knijnenburg; and Heather Richter Lipford (2017). Making privacy personal: Profiling social network users to inform privacy education and nudging. International Journal of Human-Computer Studies, vol. 98, pp. 95-108.

Woodruff, Allison; Vasyl Pihur; Sunny Consolvo; Laura Brandimarte; and Alessandro Acquisti (2014). Would a Privacy Fundamentalist Sell Their DNA for $\$ 1000 \ldots$ If Nothing Bad Happened as a Result? The Westin Categories, Behavioral Intentions, and Consequences. In SOUPS 2014. Proceedings of the 10th Symposium On Usable Privacy and Security, Menlo Park, CA, USA, 9-11 July, 2014. USENIX Association, pp. 1-18.

Yang, Hongwei (2013). Young American consumers' online privacy concerns, trust, risk, social media use, and regulatory support. Journal of New Communications Research, vol. 5, no. 1, pp. $1-30$.

Yang, Kai-Cheng; Onur Varol; Clayton A Davis; Emilio Ferrara; Alessandro Flammini; and Filippo Menczer (2019). Arming the public with artificial intelligence to counter social bots. Human Behavior and Emerging Technologies, vol. 1, no. 1, pp. 48-61.

Yaqub, Ussama; Soon Ae Chun; Vijayalakshmi Atluri; and Jaideep Vaidya (2017). Analysis of political discourse on twitter in the context of the 2016 US presidential elections. Government Information Quarterly, vol. 34, no. 4, pp. 613-626.

Yeşiltaş, Gökçe; and Tunga Güngör (2020). Intrinsic and Extrinsic Evaluation of Word Embedding Models. In ASYU 2020. Proceedings of the 2020 Innovations in Intelligent Systems and Applications Conference, Istanbul, Turkey, 15-17 October, 2020. pp. 1-6.

Yun, Haejung; Gwanhoo Lee; and Dan J Kim (2019). A chronological review of empirical research on personal information privacy concerns: An analysis of contexts and research constructs. Information \& Management, vol. 56, no. 4, pp. 570-601.

Zarifis, Alex; Richard Ingham; and Julia Kroenung (2019). Exploring the language of the sharing economy: Building trust and reducing privacy concern on Airbnb in German and English. Cogent Business \& Management, vol. 6, no. 1, article 1666641. 
Zhao, Jieyu; Yichao Zhou; Zeyu Li; Wei Wang; and Kai-Wei Chang (2018). Learning GenderNeutral Word Embeddings. In EMNLP 2018. Proceedings of the 2018 Conference on Empirical Methods in Natural Language Processing, Brussels, Belgium, 31 October - 4 November, 2018. Association for Computational Linguistics, pp. 4847-4853.

Zou, Yixin; Abraham H. Mhaidli; Austin McCall; and Florian Schaub (2018). "I've Got Nothing to Lose": Consumers' Risk Perceptions and Protective Actions after the Equifax Data Breach. In SOUPS 2018. Fourteenth Symposium on Usable Privacy and Security, Baltimore, MD, USA, 12-14 August, 2018. Baltimore, MD: USENIX Association, pp. 197-216.

Zukowski, Tomasz; and Irwin Brown (2007). Examining the influence of demographic factors on internet users' information privacy concerns. In SAICSIT '07. Proceedings of the 2007 Annual Conference of the South African Institute of Computer Scientists and Information Technologists, Port Elizabeth, South Africa, 2-3 October, 200\%. New York, NY, USA: Association for Computing Machinery, pp. 197-204.

Šidák, Zbyněk (1967). Rectangular Confidence Regions for the Means of Multivariate Normal Distributions. Journal of the American Statistical Association, vol. 62, no. 318, pp. 626-633. 